\title{
Noise of High-Performance Aircraft at Afterburner
}

\author{
Christopher K. W. Tam and Sarah A. Parrish \\ Florida State University, Tallahassee, FL 32306-4510, USA \\ Email: tam@math.fsu.edu
}

The noise from a high-performance aircraft at afterburner is investigated. The main objective is to determine whether the dominant noise components are the same or similar to those of a hot supersonic laboratory jet. For this purpose, measured noise data from F-22A Raptors are analyzed. It is found, based on both spectral and directivity data, that there is a new dominant noise component in addition to the usual turbulent mixing noise. The characteristic features of the new noise component are identified. Measured data indicates that the new noise component is observed only when the rate of fuel burn of the engine is increased significantly above that of the intermediate power setting. This suggests that the new noise component is combustion related. The possibility that it is indirect combustion noise generated by the passage of hot spots from the afterburner through the nozzle of the jet is investigated. Because flow and temperature data were not measured in the $\mathrm{F}-22 \mathrm{~A}$ engine tests, to provide support to the proposition, numerical simulations of indirect combustion noise generation due to the passing of an entropy wave pulse (a hot spot) through a military-style nozzle are carried out. Sound generation is observed at the front and at the back of the pulse. This creates a fast and a slow acoustic wave as the sound radiates out from the nozzle exit. Quantitative estimates of the principal directions of acoustic radiation due to the emitted fast and slow acoustic waves are made. It is found that there are reasonably good agreements with measured data. To estimate the intensity level (IL) of the radiated indirect combustion noise, a time-periodic entropy wave train of $15 \%$ temperature fluctuation is used as a model of the hot spots coming out of the afterburner. This yields an IL of $175.5 \mathrm{~dB}$. This is a fairly intense noise source, well capable of causing the radiation of the new jet noise component.

\section{Introduction}

It is known that the dominant noise components of a hot supersonic laboratory jet are turbulent mixing noise and shock cell noise. Figure 1 shows an F-22 high-performance aircraft tied down for testing with one engine operating with the afterburner on and the other engine at idle. The radiance from the jet plume is clearly seen. Also (barely) visible is a row of shock cells. The present investigation began with the question, "Does a jet at afterburner have the same dominant noise components as a hot supersonic laboratory jet?" As far as is known, this question has not been addressed in the open literature. This paper reports a first attempt to determine whether or not jets at afterburner condition have any new dominant noise components. This work is exploratory in nature. It must be emphasized that only a few sets (some are not complete sets) of noise data on jets of this type are published in the open literature. There are no accompanying jet flow and temperature measurements. So the results of the data analysis, as well as theoretical modeling and solutions to the model problems, reported in this paper merely represent our best effort to provide an answer to the above question. 


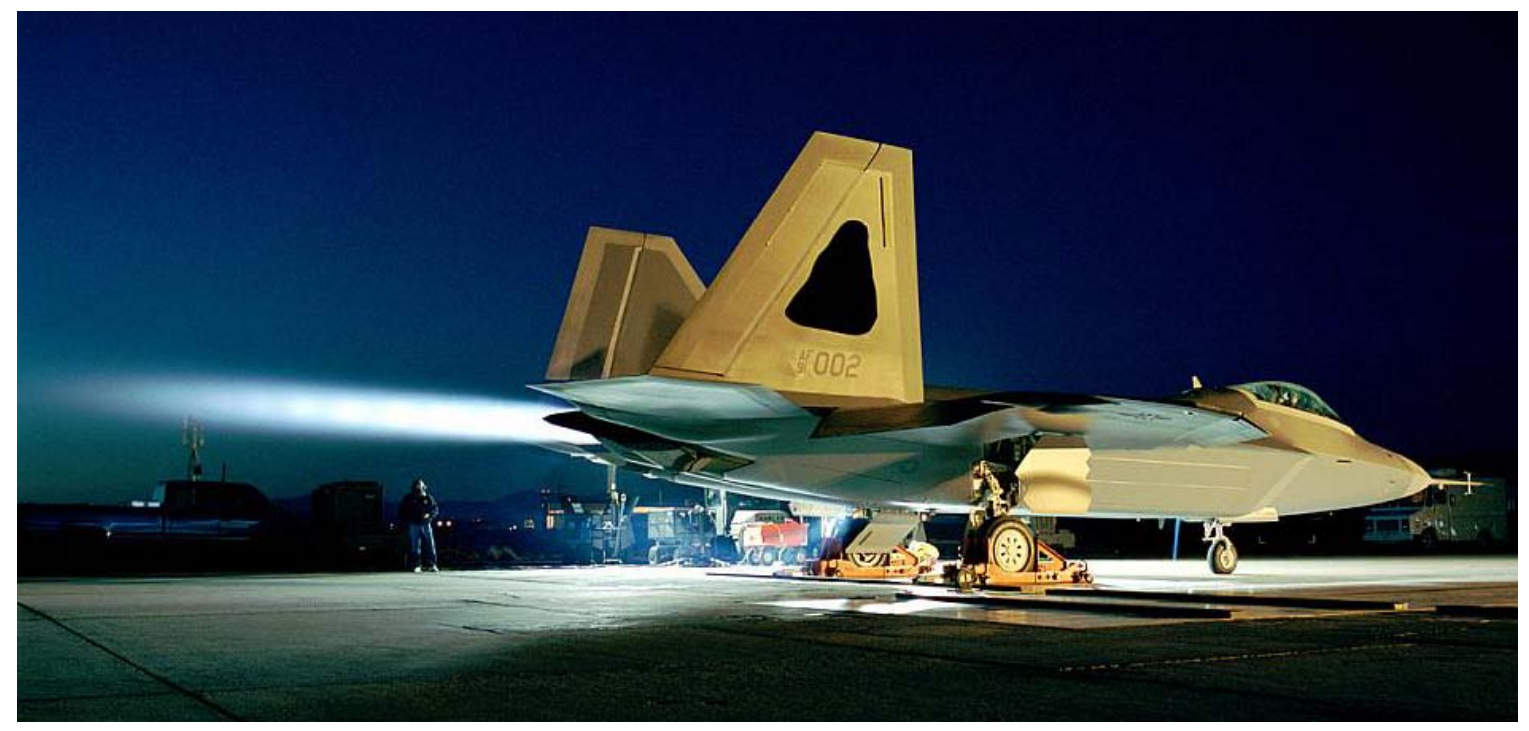

Figure 1. An F-22 Raptor aircraft tied down for testing.

This investigation has two main objectives. They are:

1. To provide credible evidence that at afterburner condition, the dominant noise components of a military-style jet are not the same as those of a standard laboratory hot supersonic jet.

2. To propose plausible noise generation mechanisms for the new noise components.

There are very few data sets in the open literature on the noise of high-performance aircrafts at afterburner condition. After a diligent search, we were able to find one relatively complete set of data (Neilsen, Gee \& Wall [1]) and three other partial sets of data (Gee, Sparrow, James, Downing, Hobbs, Gabrielson \& Atchley [2], Wall, Gee, James, Bradley, McInerny \& Neilsen [3], Neilsen, Gee, James [4]) . All these data are measured in outdoor facilities. Since these are military jet noise data, they are all reported in $1 / 3$ octave band. By design, 1/3 octave band gives more weight to high-frequency noise. Thus, the $1 / 3$ octave band spectral shape is somewhat distorted. To eliminate this bias, all the data are first converted to narrow band before they are analyzed.

Regardless of whether a jet operates with the afterburner on or not, the gas of the jet will undergo turbulent mixing with the ambient gas. Therefore, turbulent mixing noise will be emitted from the jet plume. In the presence of shock cells, the passage of large turbulence structures through the shock cells will lead to shock-turbulence interaction. This will result in the radiation of broadband shock cell noise. So it is important to acknowledge that military-style jets operating at afterburner condition will radiate turbulent mixing noise as well as shock cell noise. The question of interest is whether these jets radiate additional noise that is as dominant or even more dominant than the turbulent mixing noise and broadband shock cell noise. To identify any new noise components, it is first necessary to identify the known noise components in a laboratory jet noise spectrum. For this purpose, we use the known characteristics of these two noise components. To facilitate this process, we will briefly review the principal characteristics of both turbulent mixing noise and shock cell noise. 
According to the two-source model theory of turbulent mixing noise (see Tam [5], Tam, Golebiowski \& Seiner [6], Tam, Vishwanathan, Ahuja \& Panda [7], Suzuki [8]), the turbulence in the mixing layer of a jet consists of fine-scale turbulence and large turbulence structures. The two types of turbulent motion coexist in the jet. Both radiate noise, but with vastly different characteristics. After a thorough analysis of an extensive collection of the NASA Langley Research Center Jet Noise Laboratory data, Tam, Golebiowski, and Seiner [6] found empirically two seemingly universal spectra that were able to fit all the jet turbulent mixing noise spectra in the data bank. This is true regardless of jet Mach number and jet temperature. (Note: The highest temperature ratio in the data set is 4.9. This temperature ratio should be comparable to that of military-style jets at afterburner). Figure 2 shows the two similarity spectra plotted as functions of $f / f_{\text {peak }}$, where $f$ is the frequency and $f_{\text {peak }}$ is the frequency at the peak of the spectrum. The $F$ spectrum, or the peaky spectrum, fits all noise spectra measured in the downstream directions within a cone around the jet axis. The $G$-spectrum, or the broad spectrum, fits all the measured noise spectra radiated in the upstream and sideline directions. In the transitional directions, a superposition of the contributions from the two spectra is needed to fit the measured spectra. Tam et al [6] identified the peaky spectrum to be the noise spectrum from the large turbulence structures and the broad spectrum to be that of the noise from the fine-scale turbulence. The relationship between noise sources and sound field is shown in Fig. 3.

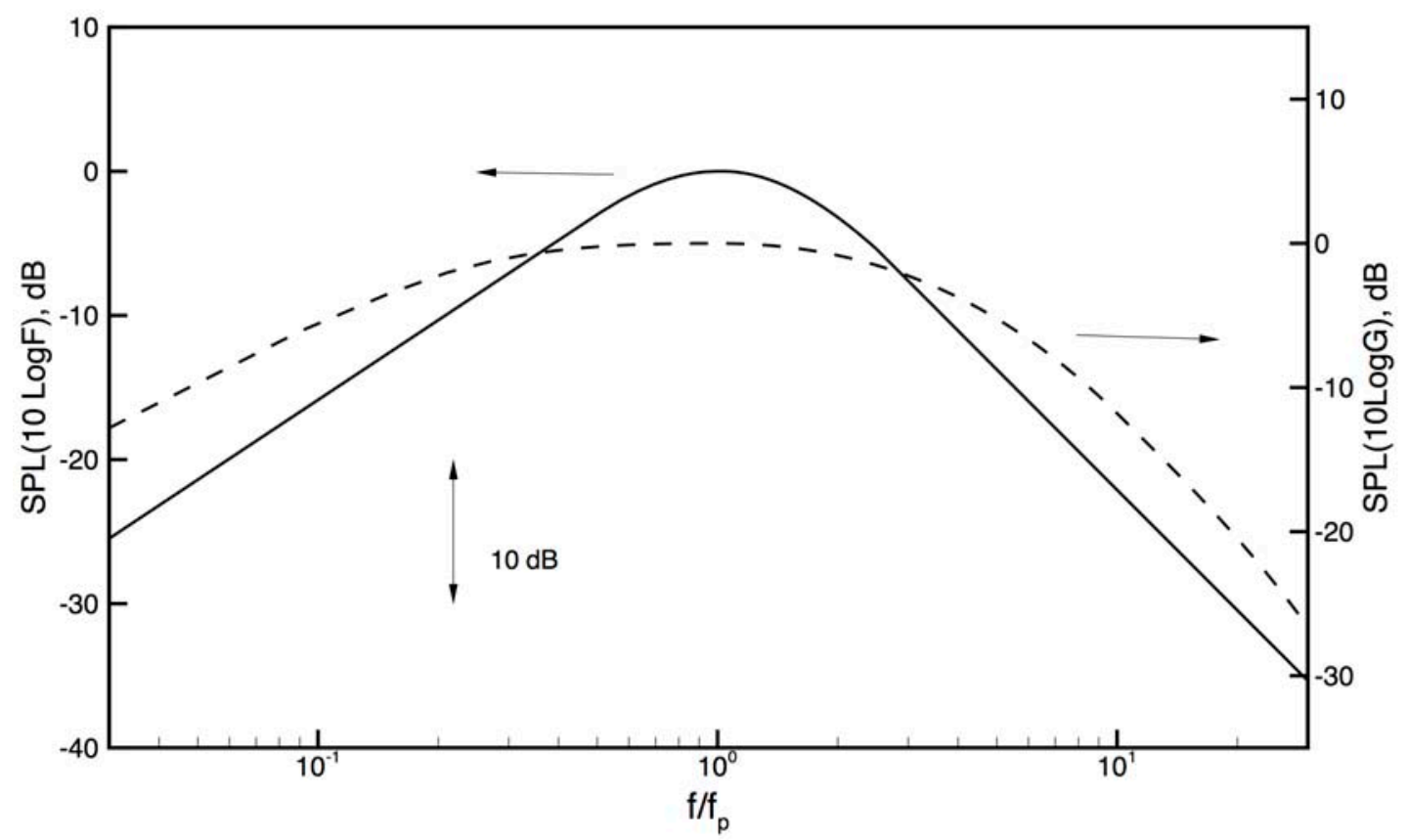

Figure 2. Similarity spectra for the two components of turbulent mixing noise: $\ldots$, large turbulence structures noise; - - - , fine-scale turbulence noise. 


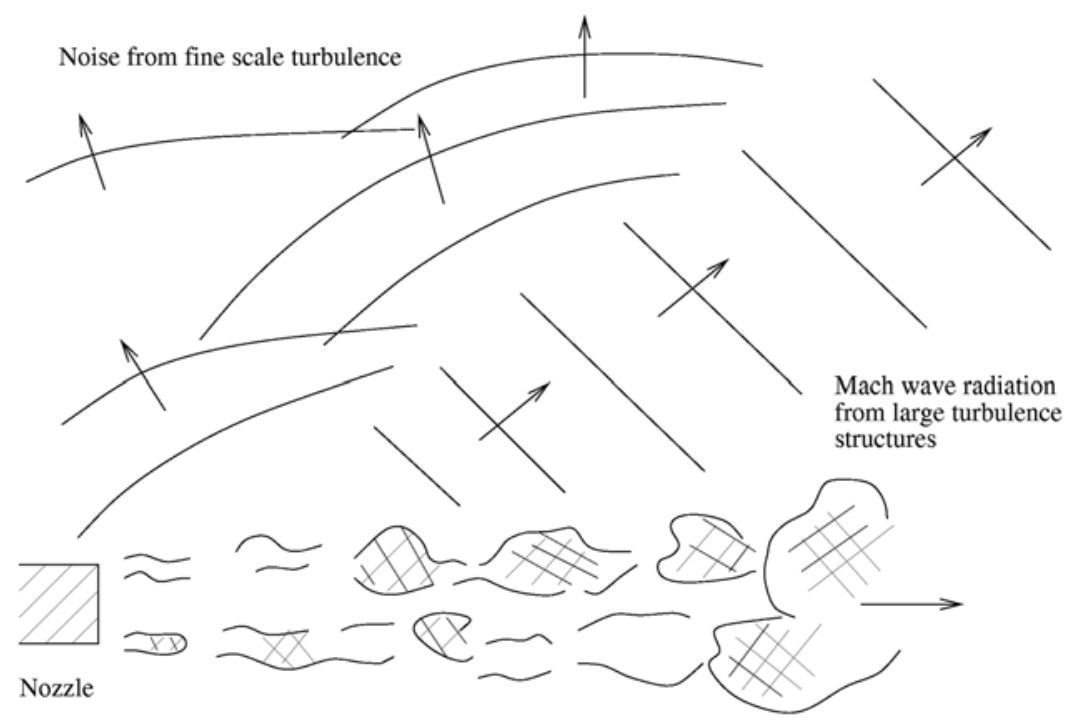

Figure 3. Schematic diagram showing the large turbulence structures of a high-speed jet, the sound field from the fine-scale turbulence, and the Mach wave radiation from the large turbulence structures.

As a demonstration of how well the similarity spectra fit measured noise data, figure 4 shows the far-field noise spectra, normalized to a distance of 100 fully expanded jet diameters, from three hot jets at Mach numbers 0.5, 1.0, and 2.0, at a temperature ratio of 3.2. The data are from Seiner, Ponton, Jansen \& Lagen [9] and Viswanathan [10]. Figures 4a and 4b show how well the $G$-spectrum fits the data at inlet angles $90^{\circ}$ and $100^{\circ}$. The fitting is done by positioning the peak of the similarity spectrum to coincide with that of the measured data. Figures $4 \mathrm{e}$ and $4 \mathrm{f}$ show how well the $F$-spectrum fits the measured data at the $130^{\circ}$ and $140^{\circ}$ directions. In the transitional directions, at $110^{\circ}$ and $120^{\circ}$, a combination of the two similarity spectra is required to produce a spectrum that fits the measurement. This is shown in Figs. 4c and 4d. The similarity spectra will be used later in this paper to identify the turbulent mixing noise components in military-style jet noise spectra. We would like to emphasize that large turbulence structures noise is the dominant noise component at the $130^{\circ}$ and $140^{\circ}$ directions. This observation will be used later.

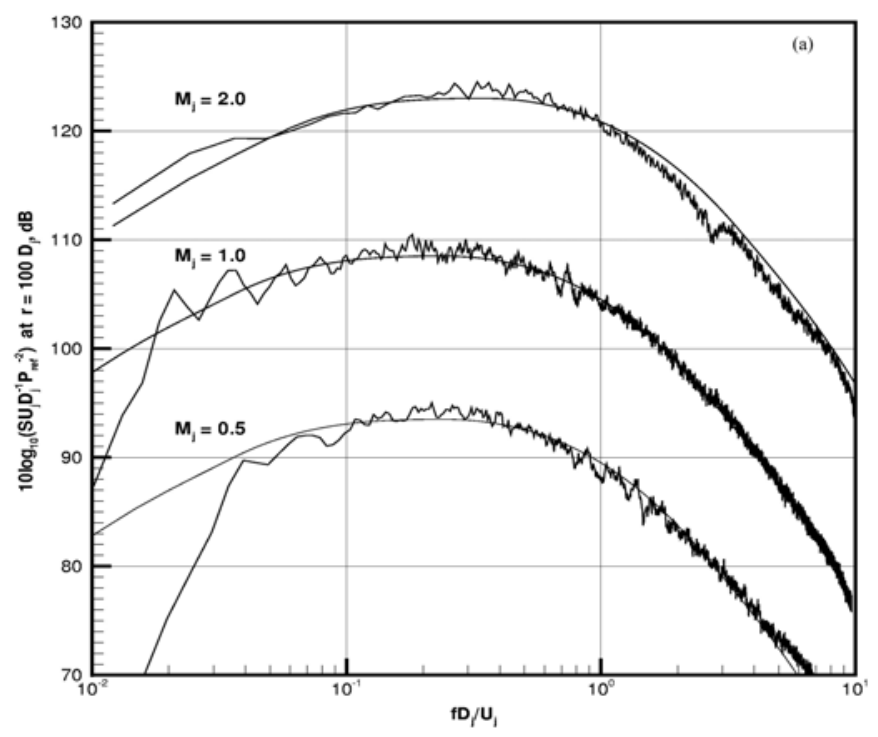



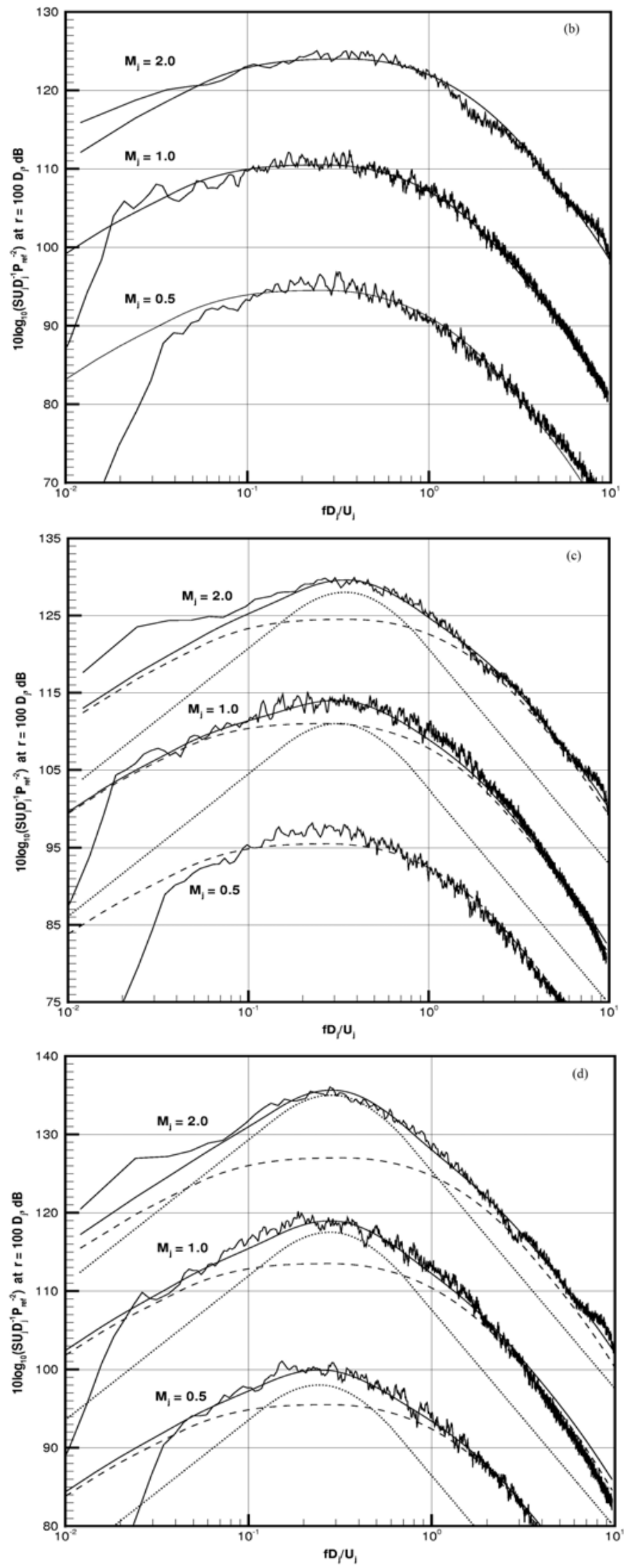

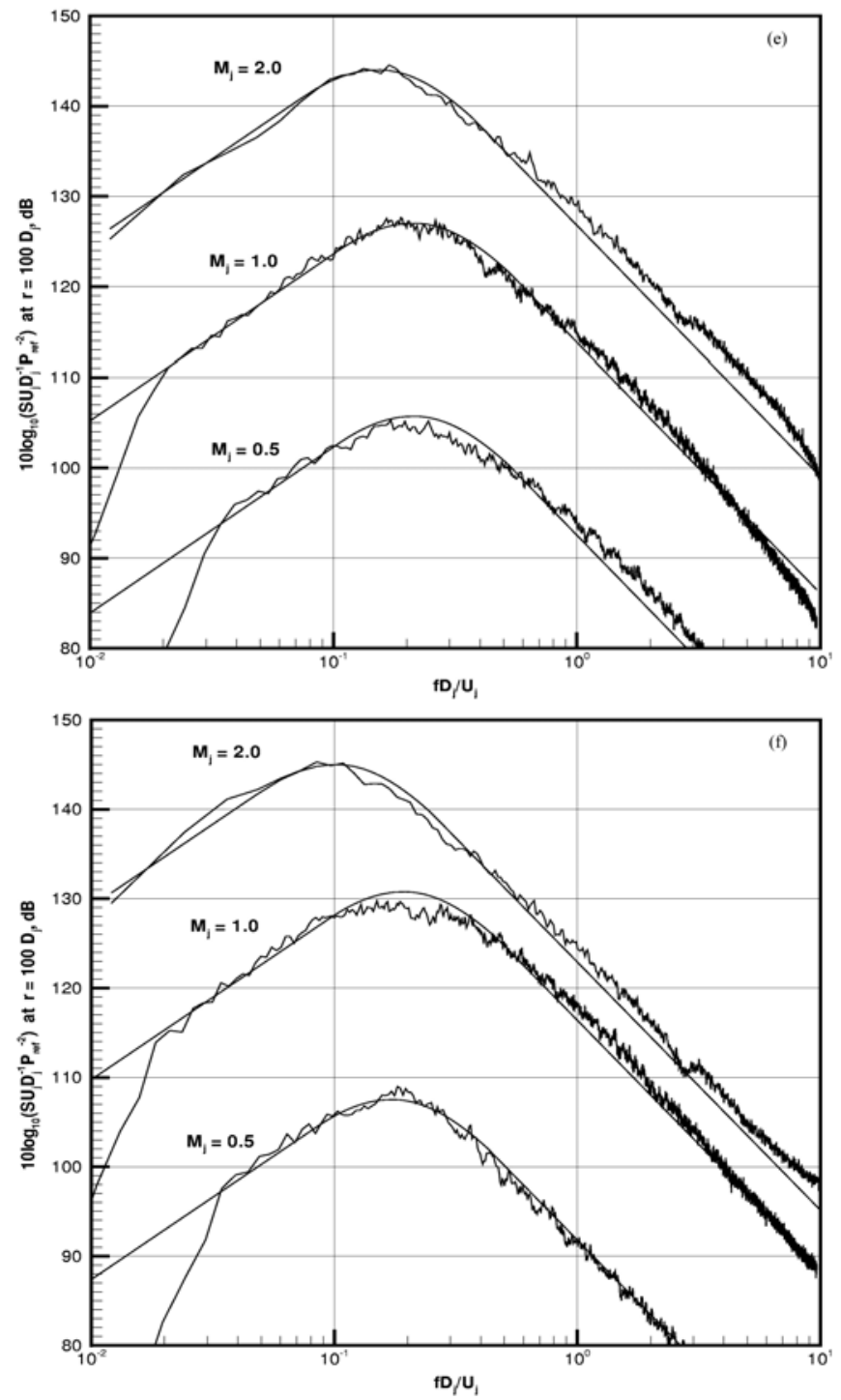

Figure 4. Comparisons between similarity spectra (smooth curves) and measured spectra by Seiner et al. [9] and Viswanathan [10]; $T_{\mathrm{r}} / T_{\mathrm{a}}=3.2$; inlet angle (a) $90^{\circ}$, (b) $100^{\circ}$, (c) $110^{\circ}$, (d) $120^{\circ}$, (e) $130^{\circ}$, (f) $140^{\circ}$.

Of the two turbulent mixing noise components of high-speed jets, the large turbulence structures noise is more dominant. The noise is Mach wave radiation emanated directly from the large turbulence structures (see figure 3) and is confined to a Mach cone in the downstream direction around the jet axis. The fine-scale turbulence noise, on the other hand, radiates in all directions and is dominant in the upstream and sideline directions, where there is no Mach wave radiation. Within the Mach cone, fine-scale turbulence noise is the subdominant component and is, therefore, not generally observed. 


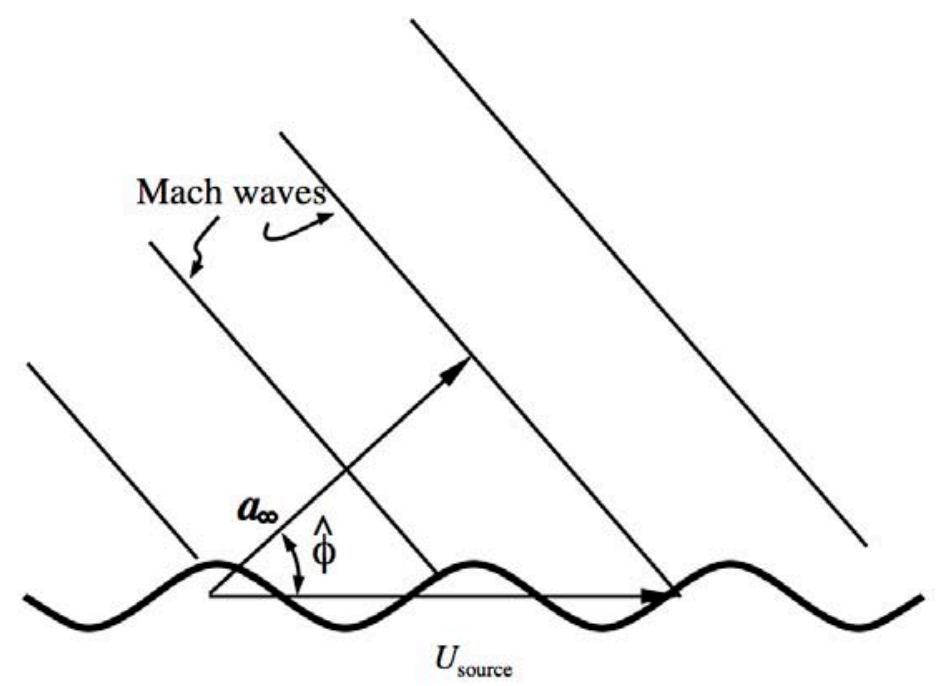

Figure 5. Velocity triangle of Mach wave radiation from a supersonically moving wavy source.

To understand why the noise from the large turbulence structures radiates as Mach waves, one may regard these relatively coherent structures as a supersonically traveling wavy wall. Figure 5 shows the velocity triangle of Mach wave radiation from such a moving source. The angular direction of Mach wave radiation, $\hat{\phi}$, (measured from the flow direction; exhaust angle) is related to the propagation speed, $U_{\text {source, }}$ of the large turbulence structures by the relation,

$$
\hat{\phi}=\cos ^{-1}\left(a_{\infty} / U_{\text {source }}\right)
$$

where $a_{\infty}$ is the ambient speed of sound. According to (1), $\hat{\phi}$ increases with an increase in propagation speed. For a low supersonic jet (Mach number less than 1.5), a fairly good approximation is to take $U_{\text {source }}$ to be $0.7 U_{\text {jet }}$ (where $U_{\text {jet }}$ is the fully expanded jet velocity). For a high supersonic jet (jet Mach number near and above 2) the use of $U_{\text {source }} \approx 0.8 U_{j}$ is found preferable. The dominant direction of noise radiation of a supersonic jet may be found by means of equation (1). As a confirmation that Mach wave radiation plays an important role in the noise of high-speed jets, Tam [11] compared the radiation direction given by equation (1) with the directions of maximum noise radiation from the data of three Mach 1.92 jets measured by Petitjean, Viswanathan, and McLaughlin [12]. The temperature ratios $\left(T_{\text {jet }} / T_{\text {ambient }}\right)$ of the jets were $0.58,1.32$, and 1.65 . The results are shown in figure 6 . It is easy to see that the predicted directions (indicated by arrows) agree well with measurements. Later in this paper, we will use this method to determine the directions of radiation of the dominant noise components of highperformance jets at afterburner. 


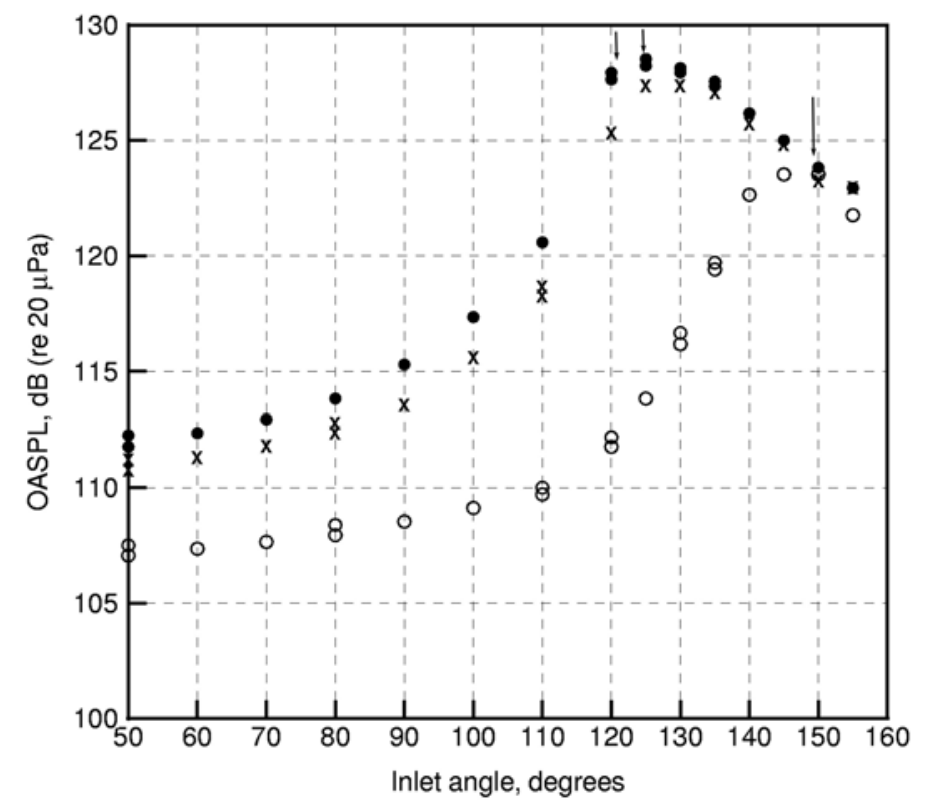

Figure 6. OASPL directivity pattern of a Mach 1.92 jet at $T_{\text {jet }} / T_{\text {ambient }}=0.58$ (open circles), 1.32 (crosses), and 1.65 (filled circles). Data from Petitjean et al. [12].

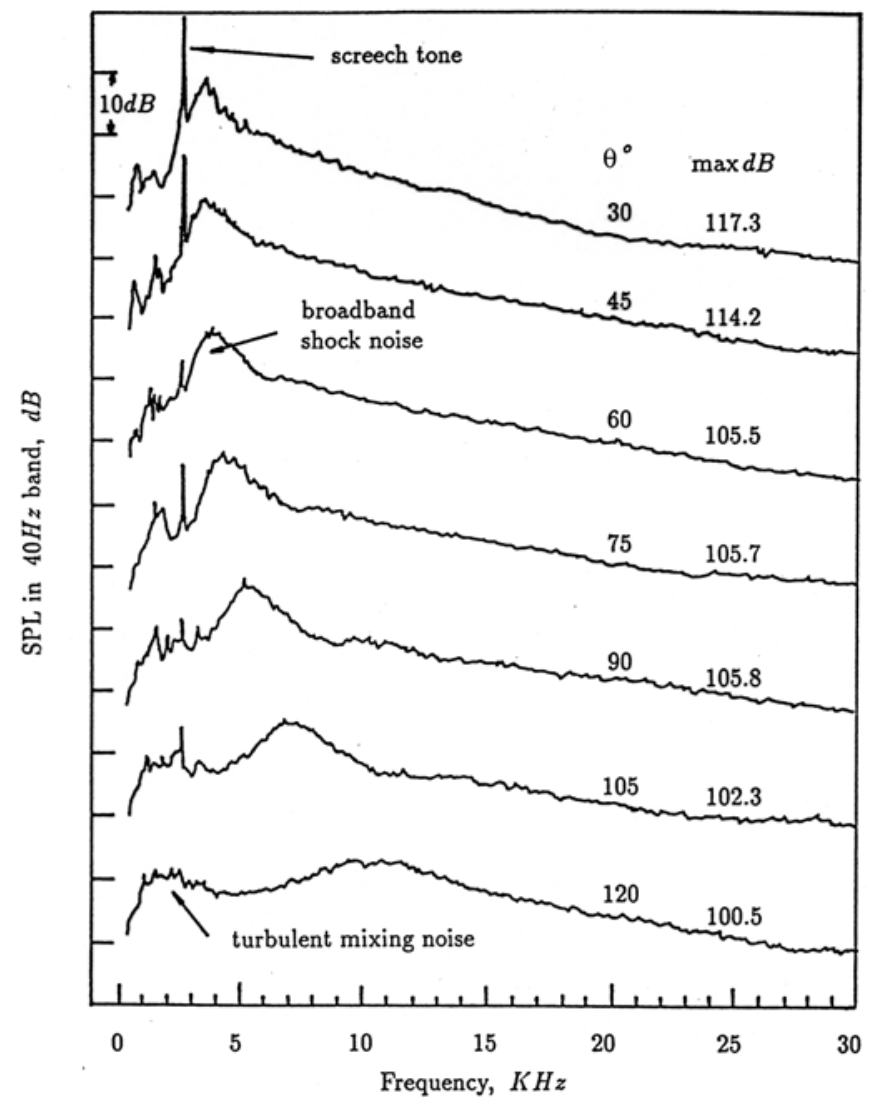

Figure 7. Narrow band noise spectra of a Mach 1.67 jet from a convergent-divergent nozzle with design Mach number 1.5. $\theta$ (inlet angle) is the direction of radiation. Data from Norum and Seiner [14]. 
It is known that the peak direction of radiation of broadband shock cell noise depends strongly on frequency (Tam and Tanna [13]). Such dependence can be easily seen in the measurements of Norum and Seiner [14] shown in figure 7. In this figure, the relatively narrow peak is the broadband shock cell noise. Notice that the frequency at the spectral peak increases with an increase in inlet angle $\theta$, the direction of radiation. The maximum level of SPL in each spectrum is provided on the right side of the figure. Based on these levels, it is clear that the direction of most intense shock cell noise radiation is at small angles $\theta$ in the upstream direction.

The rest of this paper is as follows. In section 2, an analysis of the F-22A aircraft noise data is presented. It will be shown that as the rate of engine fuel burn increases, a new noise component emerges in the noise data. At afterburner condition, the new noise component is dominant over the angular sector from $\theta=110$ degrees to 130 degrees and for frequencies above $200 \mathrm{~Hz}$. In section 3, a plausible generation mechanism for the new noise component is proposed. Because of the scarcity of available data, a definitive confirmation is not possible at this time. For this reason, it is important to regard the proposed mechanism as merely a likely mechanism. It is believed that the new noise component is combustion related. Unlike laboratory jet mixing noise, the proposed noise component is generated inside the nozzle of the jet and not in the jet plume. Results of numerical simulations will be presented to demonstrate that the passage of hot spots (entropy waves) through a military-style nozzle can, indeed, lead to the generation of intense noise. This is generally referred to as indirect combustion noise. Here, it is proposed as the source of the new noise component. In the absence of measured flow and temperature fluctuation data, numerical simulation results are the best we can offer to support the proposed noise mechanism. Section 4 summarizes the findings of this investigation. Conclusions are provided.

\section{Data Analysis}

\subsection{Source of data}

The main data set used in the present investigation is the F-22A jet noise data at afterburner published by Neilsen, Gee and Wall [1]. Other than this set of data, noise data from Gee, Sparrow, James, Downing, Hobbs, Gabrielson and Atchley [2], Wall, Gee, James, Bradley, McInerny and Neilsen [3], Neilsen, Gee, James [4] will also be used. Figure 8 is a schematic diagram of how the data of Ref. [1] was measured. A row of microphones was placed in a straight line 11.7 meters from the jet centerline. The point marked by a cross in figure 8 was determined as the apparent center of noise radiation by the previous works of the authors of Neilsen, Gee and Wall [1] and colleagues. The directivity of the measured data covers the angular range of $60^{\circ}$ to $150^{\circ}$ inlet angle at $10^{\circ}$ intervals. The published data is in $1 / 3$ octave band. 


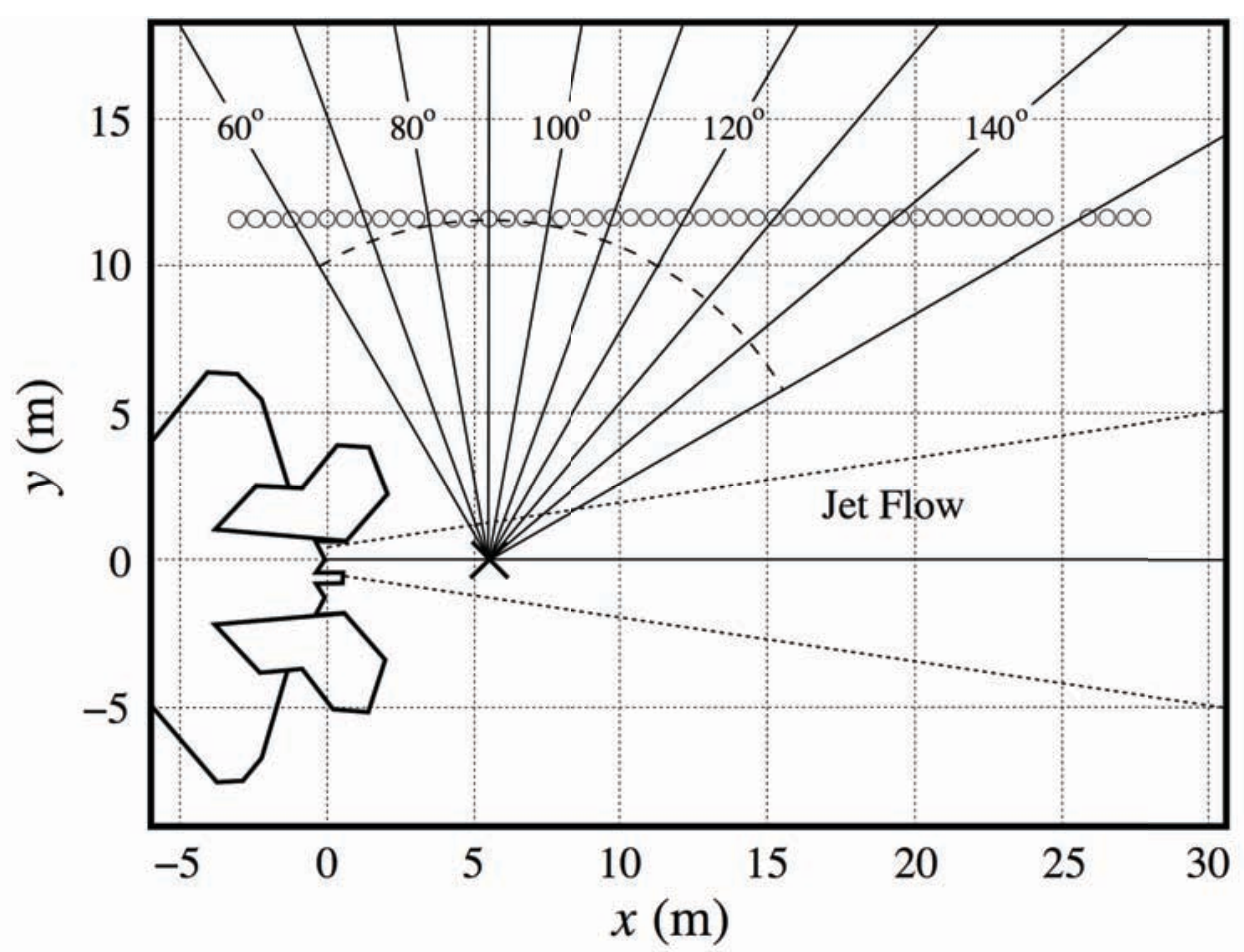

Figure 8. Schematic diagram showing the position of a row of microphones (circles) used to measure the jet noise data. The cross is regarded as the location of the apparent center of jet noise by the authors and coworkers of Neilsen, Gee and Wall [1].

The data of Neilsen, Gee \& Wall [1] as published is not in a form suitable for analysis. The following operations are, therefore, performed on the data set. First, the data is converted to narrow band (per $\mathrm{Hz}$ in $\mathrm{dB}$ ) to remove the unnatural higher weighting given to the high-frequency components of the $1 / 3$ octave spectra. On assuming that the noise level is approximately constant over a $1 / 3$ octave band, it is straightforward to derive the following conversion formula.

$S P L($ per Hz in $\mathrm{dB})=S P(1 / 3$ octave band in $\mathrm{dB})-10 \log \left(0.2315 f_{c}\right)$

where $f_{c}$ is the center frequency in $\mathrm{Hz}$.

Second, the sound pressure level is adjusted as if each data point were measured at an equal distance from the apparent noise source center (see the circular arc in figure 8). This adjustment renders the data set ready for directivity analysis. Figure 9 shows the narrow band spectra of the noise data from inlet angles $60^{\circ}$ to $100^{\circ}$. Figure 10 shows the corresponding spectra for angular directions between $110^{\circ}$ and $150^{\circ}$. 


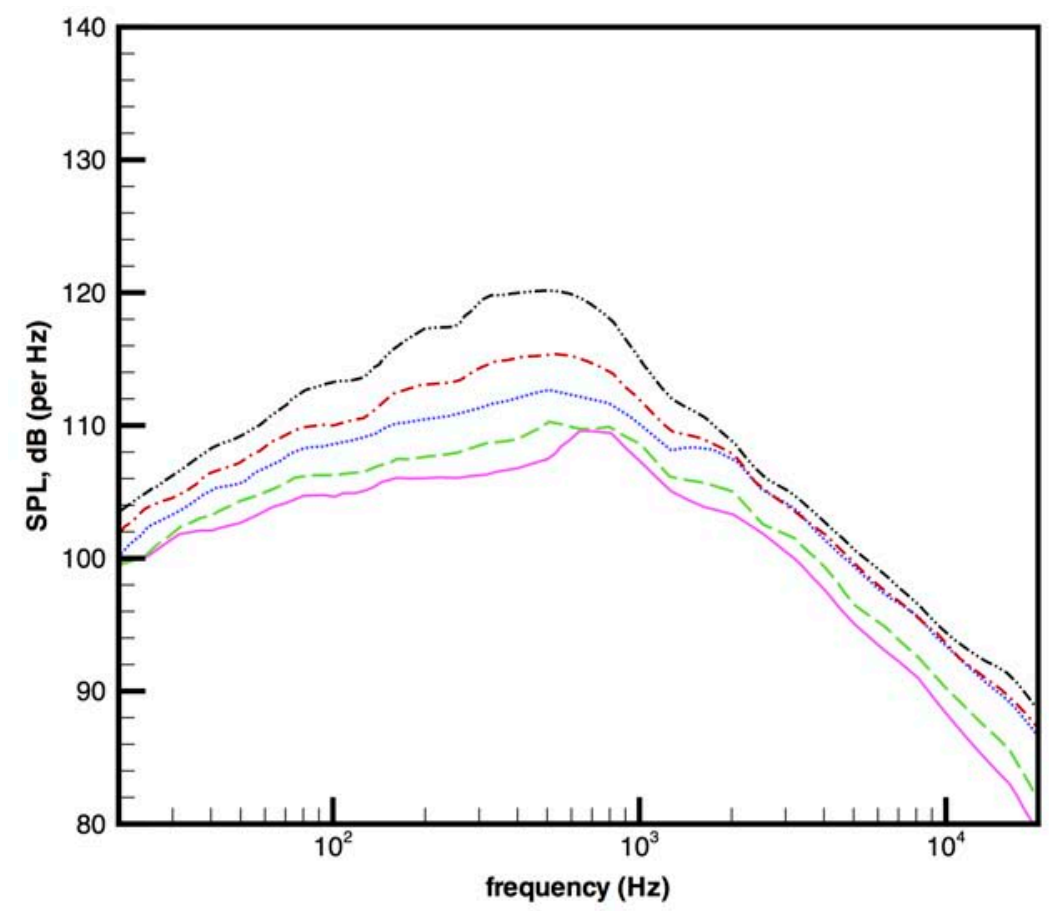

Figure 9. Narrow band F-22A noise spectrum data for inlet angles $60^{\circ}$ to $100^{\circ}$. Data from Neilsen et al $[1] .-60^{\circ},---70^{\circ}, \cdots 80^{\circ},-\cdot-90^{\circ},-\cdots-100^{\circ}$ (inlet angle).

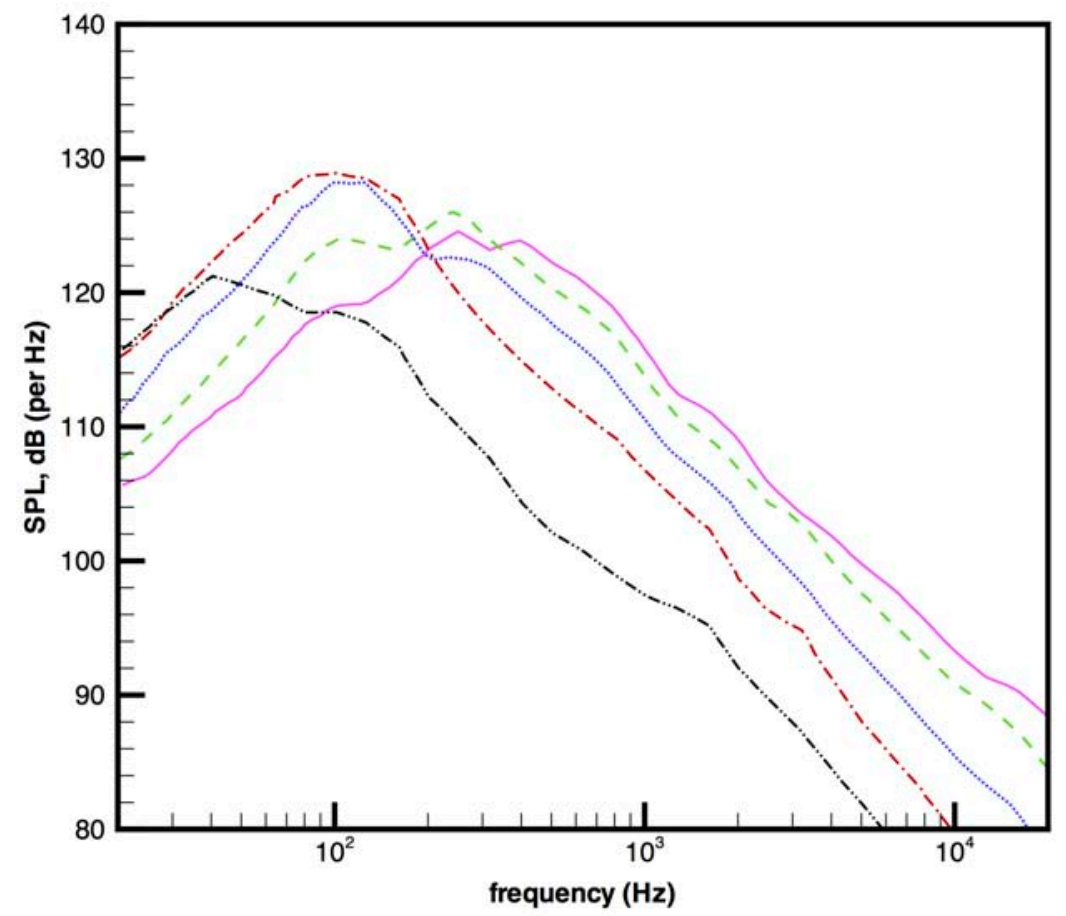

Figure 10. Narrow band F-22A noise spectrum data for inlet angles $110^{\circ}$ to $150^{\circ}$. Data from Neilsen et al $[1]-100^{\circ},---110^{\circ}, \cdots \cdots 120^{\circ},-\cdot-130^{\circ},-\cdots-140^{\circ}$ (inlet angle).

Earlier a partial set of F-22A noise data was published in Gee et al [2]. This data set was measured on a circular arc 61 meters from the jet exit at inlet angles of $115^{\circ}, 125^{\circ}, 135^{\circ}$ and 
$145^{\circ}$. Figure 11 shows the locations of the ground microphones relative to the jet exit in Gee et al's measurements. The location of the arc is far enough from the jet exit that it may be regarded as the beginning of the far field. By superimposing this set of spectra onto those of figure 10, it is easy to see that the two sets of spectra are similar and consistent. This provides confidence in the main data set used in this study. In subsection 2.3 below, the two sets of data will be used in combination for analyzing the characteristics of the F-22A aircraft noise at afterburner.

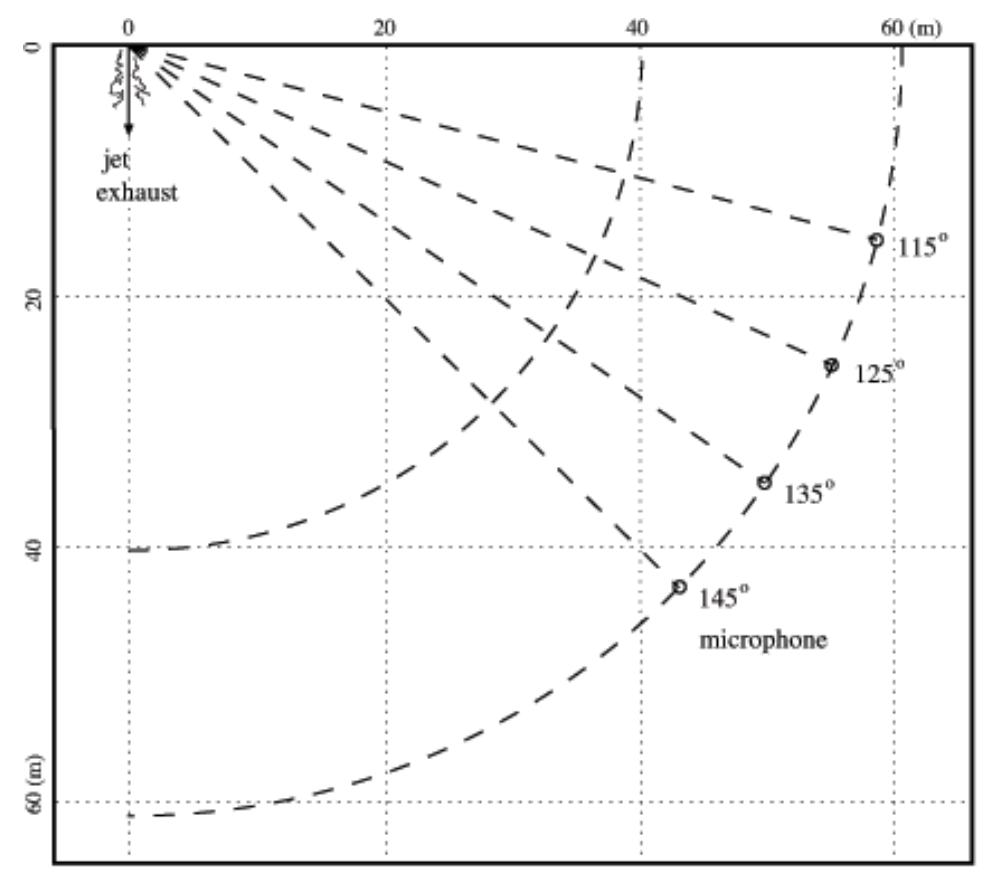

Figure 11. This figure shows the locations of the ground microphones in the Gee et al's [2] engine test measurements

Wall et al [3] provides F-22A noise spectra at three engine power settings. Even though the measured data are limited to two angles, they still demonstrate the effect of a highperformance aircraft's engine power setting on its noise characteristics. Neilsen, Gee and James [4] provides data and analysis similar to Neilsen, Gee and Wall [1].

\subsection{Noise characteristics at different engine power settings}

F-22A aircrafts usually are operated at four engine power settings. They are the idle, intermediate power, military power and afterburner. There is a direct correlation between engine power and the rate of fuel burn. At idle power, a significant component of radiated sound comes from the tones and narrow broadband peaks of turbomachinery noise. This is not of interest to the present investigation. At intermediate power, jet noise becomes the dominant noise of the engine. Noise spectral data at intermediate power, military power and afterburner at $90^{\circ}$ and $130^{\circ}$ are available in Wall et al [3]. Figure 12 shows the noise spectra in the $130^{\circ}$ direction. Obviously, the noise level increases significantly at higher power settings. The dotted curve in this figure is the similarity noise spectrum of the large turbulence structures of laboratory jets (see figure 2). The dotted curve matches the intermediate power noise spectrum very well. This suggests that at intermediate power, the noise of an F-22A aircraft is mainly turbulent mixing noise, the same as a 
laboratory jet. Figure 13 shows an attempt to compare the standard large turbulence structures noise spectrum with the measured noise spectra at military power and afterburner. The agreement is poor. This indicates that the large turbulence structures in the jet flow are somewhat different from those of standard laboratory jets. One reason is that laboratory jets are comprised entirely of gases. The jet flow of an F-22 jet at afterburner, on the other hand, is laden with fuel droplets. This affects the turbulent mixing process of the jet and consequently the radiated noise. It is to be noted that at the higher power settings the noise spectra have double peaks. This is a telltale sign that there could be more than one dominant noise component as the rate of fuel burn increases significantly.

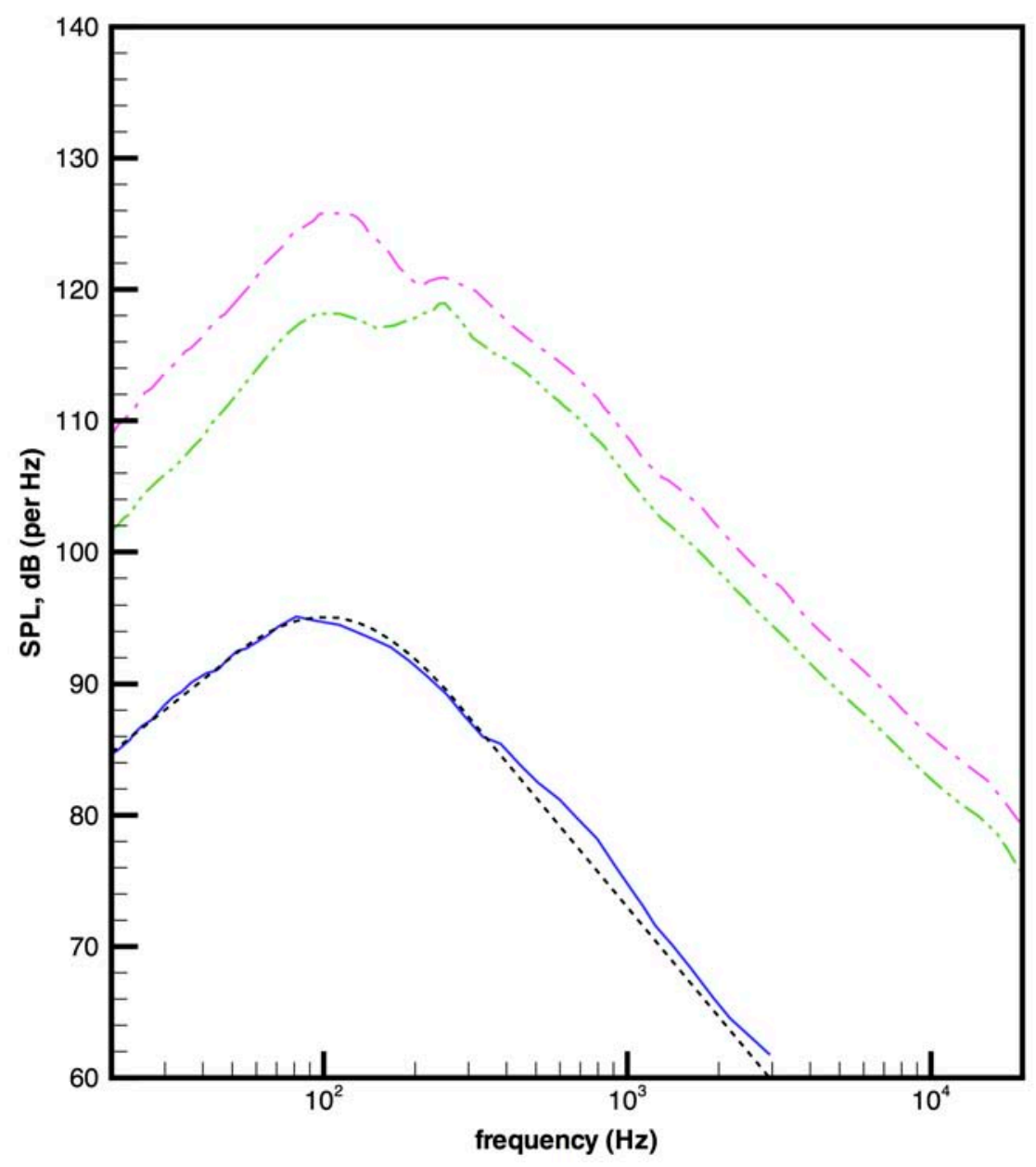

Figure 12. Noise spectra in the $130^{\circ}$ direction for three power settings. Dotted curve is the similarity spectrum of the large turbulence structures noise of laboratory jets. Data from Wall et al [3]. — intermediate power, _ - - military power, — - - afterburner. 


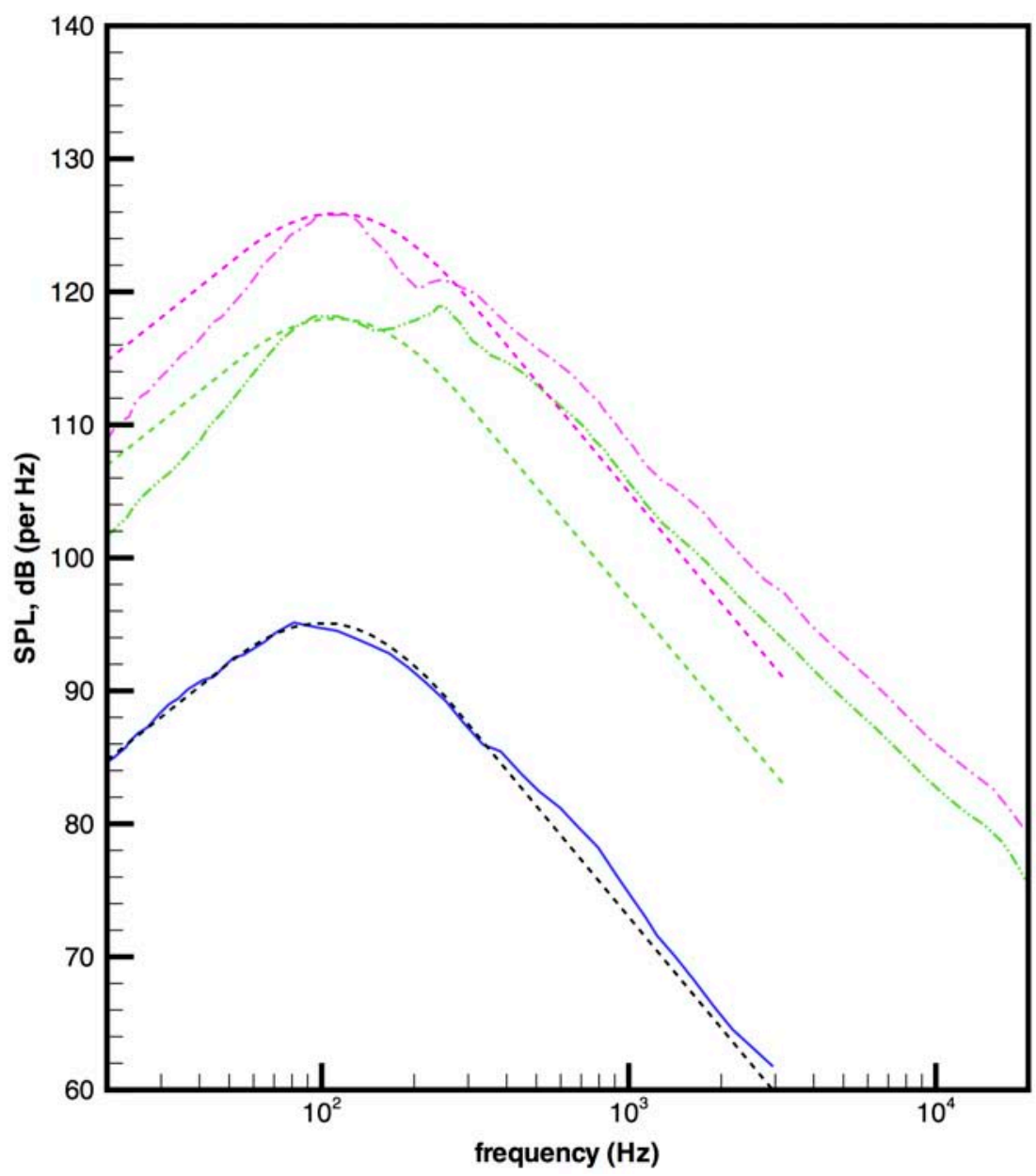

Figure 13. Noise spectra in the $130^{\circ}$ direction for three power settings. Dotted curve is the similarity spectrum of the large turbulence structures noise of laboratory jets. Data from Wall et al [3]. —— intermediate power, _ - - military power, _ - — afterburner.

Figure 14 shows the corresponding noise spectra for the three power settings at the $90^{\circ}$ direction of radiation. The dotted curve in this figure is the spectrum of the fine-scale turbulence noise from figure 2. It is a reasonably good fit for the F-22A noise spectrum at intermediate power, except for frequencies less than $100 \mathrm{~Hz}$. This provides further evidence that at intermediate power, the noise from an F-22A aircraft is similar to that of a laboratory heated supersonic jet. In figure 15, the noise spectra for the intermediate and military power settings from figure 14 are shifted up vertically in an attempt to match the spectrum for the jet at afterburner. It is readily seen that at higher power, the noise spectra are much broader in width than at intermediate power, again suggesting a change in the turbulent jet mixing noise as the rate of fuel burn increases. 


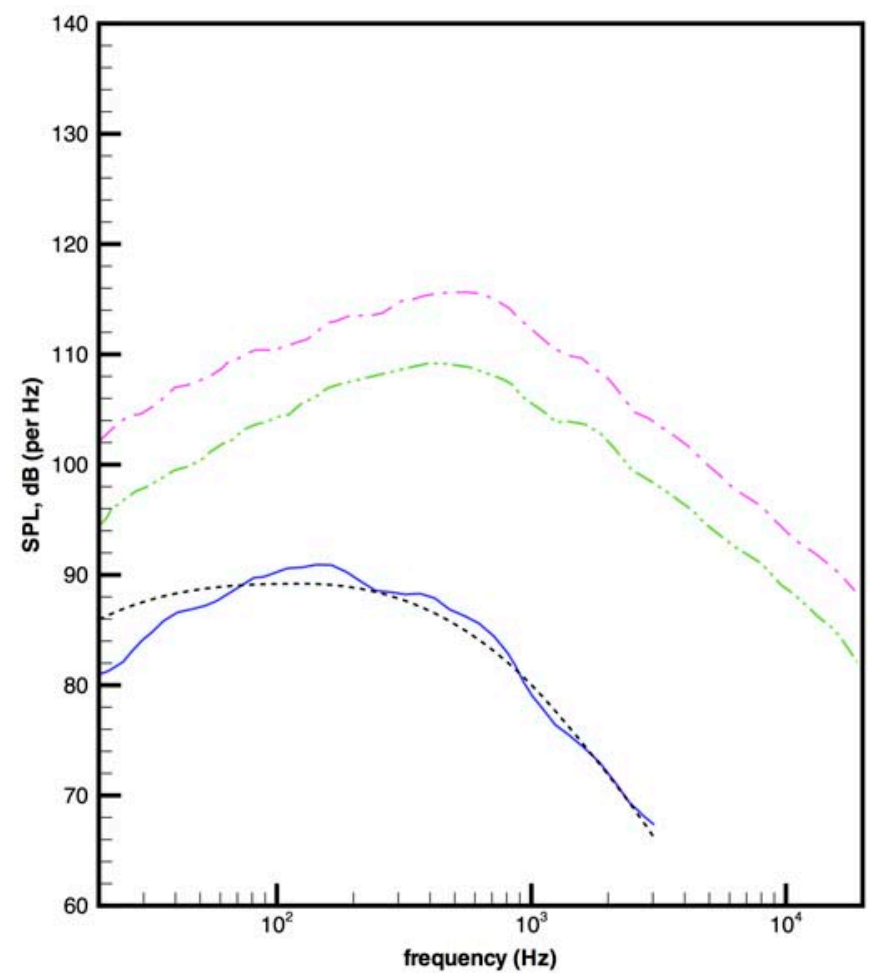

Figure 14. Noise spectra in the $90^{\circ}$ direction for three power settings. Dotted curve is the similarity spectrum of the fine-scale turbulence noise of laboratory jets. Data from Wall et al [3]. intermediate power, $-\cdots$ - military power, $-\cdots$ - afterburner.

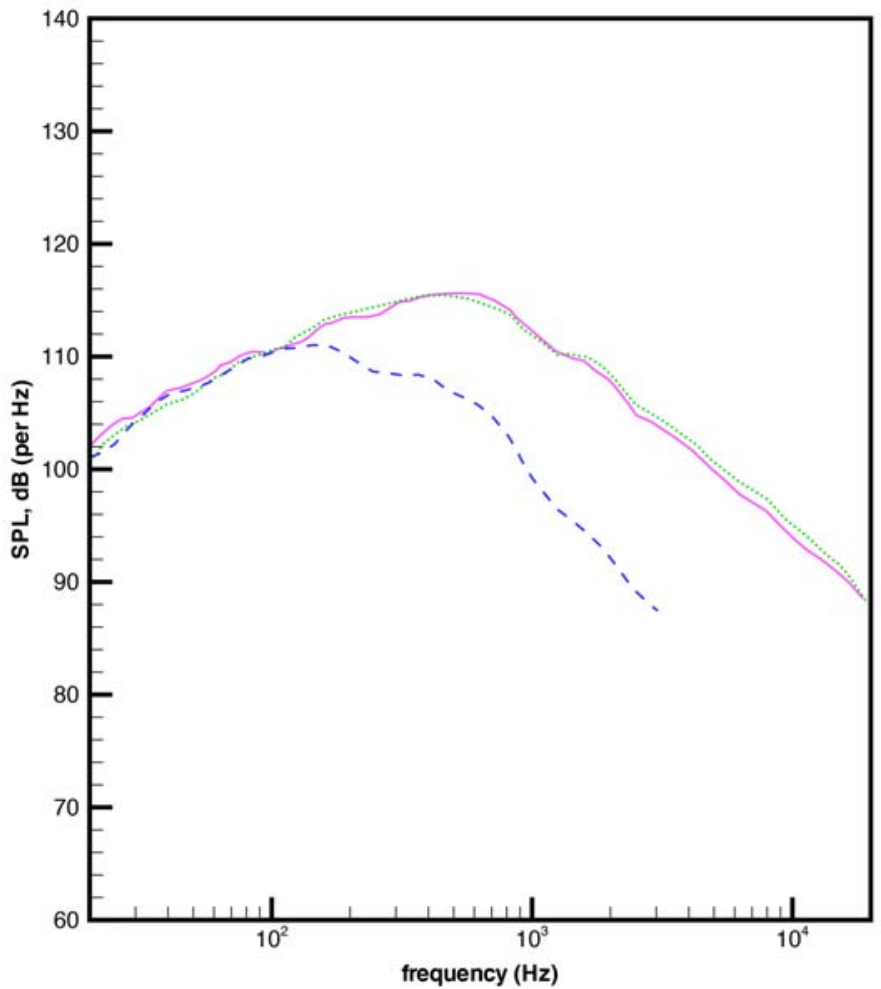

Figure 15. Noise spectra in the $90^{\circ}$ direction for three power settings. Spectra at intermediate and military power are shifted upward to match that at afterburner. Data from Wall et al [3]. - - intermediate power, $\cdots \cdots$ military power, $—$ afterburner. 


\subsection{Noise characteristics at afterburner}

To better understand the characteristics of the dominant noise components, we will examine the jet noise spectra at afterburner in figures 9 and 10, as well as the noise spectra of Gee et al [2]. From the known jet mixing noise characteristics (see Tam et al [6], [7]) we expect finescale turbulence noise to be the dominant noise component at low inlet angles. Figure 16 shows the noise spectrum at a $60^{\circ}$ inlet angle. The fine-scale turbulence noise spectrum from figure 2 is plotted in this figure as a dotted curve. It is seen that, indeed, the fine-scale turbulence noise is dominant, as evidenced by the good match between the spectrum from figure 2 and the measured data. However, in the range of 600 to $800 \mathrm{~Hz}$, there is a noise peak that is distinctly unrelated to the fine-scale turbulence noise. This peak is possibly that of a new noise component. To see how this peak evolves as a function of inlet angle, we shift the noise spectra from $60^{\circ}$ to $80^{\circ}$ upward to match the $90^{\circ}$ spectrum, as shown in figure 17. In this figure, the dotted curve is again the finescale turbulence spectrum. This figure indicates that the new narrow noise peak increases in intensity and moves to a lower frequency as inlet angle increases. This suggests that for the F$22 \mathrm{~A}$ aircraft, fine-scale turbulence noise is dominant over a broad range of frequencies in the upstream direction. However, at the sideline direction, there is a new, equally dominant noise component over a relatively narrow frequency range.

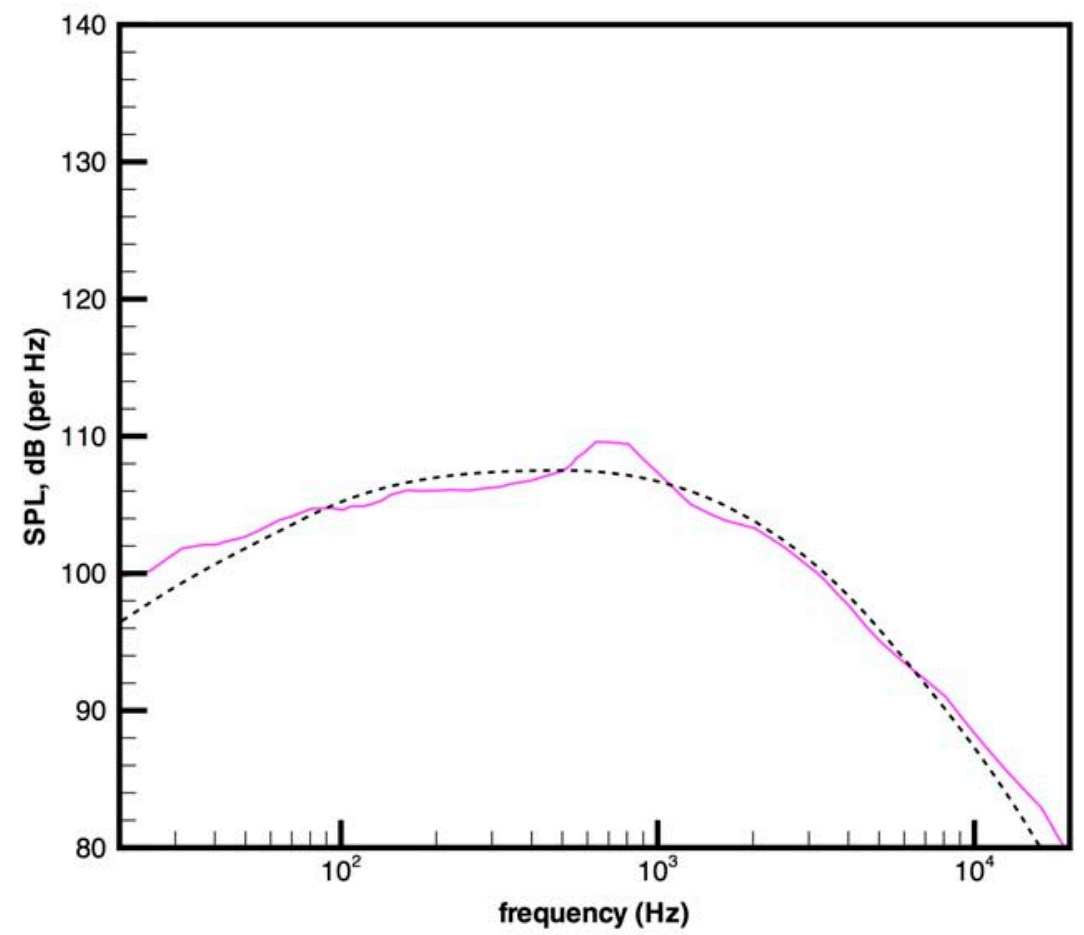

Figure 16. Noise spectrum at $60^{\circ}$ direction of radiation. Dotted curve is the fine-scale turbulence noise spectrum of laboratory jets. 


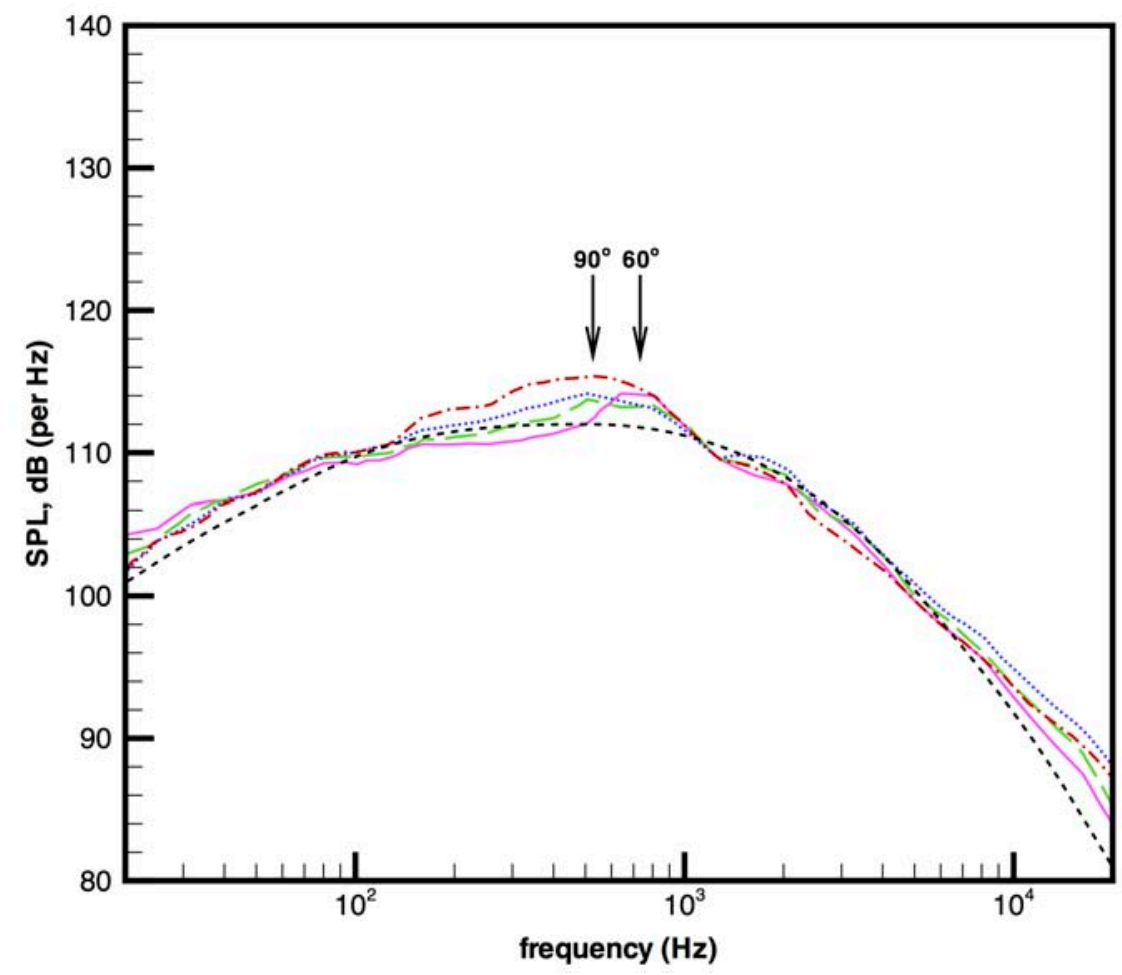

Figure 17. Noise spectra from $60^{\circ}$ to $80^{\circ}$ directions of radiation are shifted upward to match that at $90^{\circ}$. Dotted curve is the fine-scale turbulence noise spectrum of laboratory jets see figure 2). $-60^{\circ},----70^{\circ}, \cdots 80^{\circ},-\cdot-90^{\circ}$.

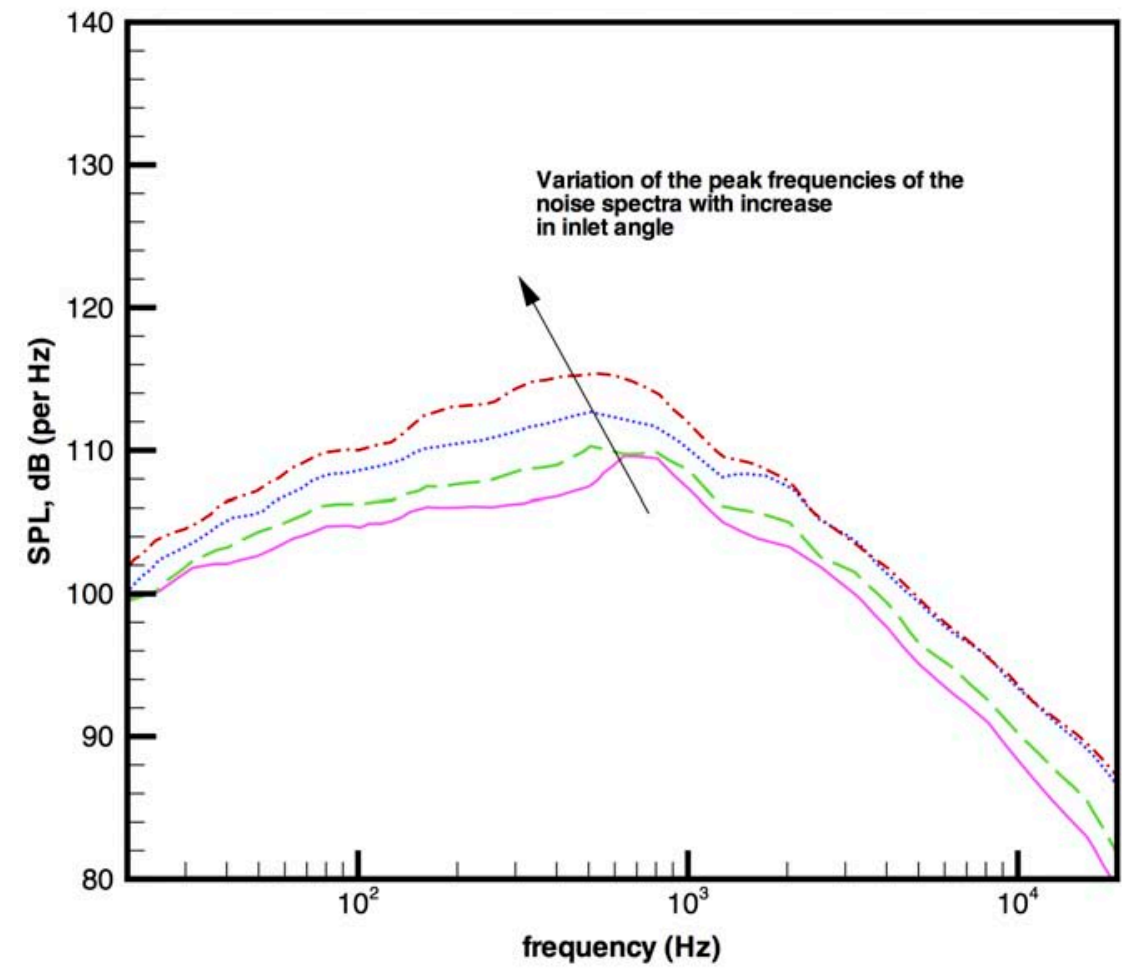

Figure 18 . Noise spectra from $60^{\circ}$ to $90^{\circ}$ reveals the increase in peak intensity and the shifting of the peak frequency to a lower frequency as inlet angle increases. $-60^{\circ},----70^{\circ}$, ... $80^{\circ},-\cdot-90^{\circ}$. 
Another way of illustrating the increasing dominance of the new noise component is shown in figure 18 . This figure shows the noise spectra from $60^{\circ}$ to $90^{\circ}$. Clearly, in this figure, the peak of the noise spectra increases in intensity and shifts to a lower frequency as inlet angle increases. The shape of the noise spectrum becomes increasingly different from that of the finescale turbulence noise. It is to be noted that the new peak in the noise spectra is not broadband shock cell noise. The new peak decreases in frequency as inlet angle increases. This is opposite to the behavior of broadband shock cell noise, which increases in frequency as inlet angle increases (see figure 7). In fact, after examining all the noise spectra, it is our conclusion that there is no evidence of broadband shock cell noise in the angular sector $60^{\circ}$ to $150^{\circ}$. However, this does not mean that broadband shock cell noise is not generated. The noise is simply buried under more dominant noise components.

It is known and was pointed out in the previous section that the most important component of turbulent mixing noise is the noise of the large turbulence structures of the jet flow. This noise component radiates primarily in the downstream direction. For a jet at afterburner, combustion takes place, not only inside the afterburner and the nozzle, but also well beyond the jet core (see figure 1). For this reason, it is plausible that the large turbulence structures of these jets are somewhat different from those of heated supersonic laboratory jets. The jet fluid is not completely gaseous but is mixed with fuel droplets. This leads to the belief that the noise spectrum may also be different, as indicated in figure 13. To identify the spectrum of the large turbulence structures noise, it appears that it would be more fruitful to investigate the noise spectra radiated to large inlet angles, as is the case for laboratory jets. Figure 19 is a superposition of the noise spectra at the $130^{\circ}$ and $140^{\circ}$ directions. The main contributions to these two spectra should come primarily from the large turbulence structures of the jet flow. This being the case, it appears that a good representation of the large turbulence structures noise spectrum is to take the part of the spectrum common to both the $130^{\circ}$ and $140^{\circ}$ spectra. This identification leads to a spectrum shape as shown in figure 20 . This spectrum will be referred to as the large turbulence structures noise spectrum of the F-22A jet at afterburner.

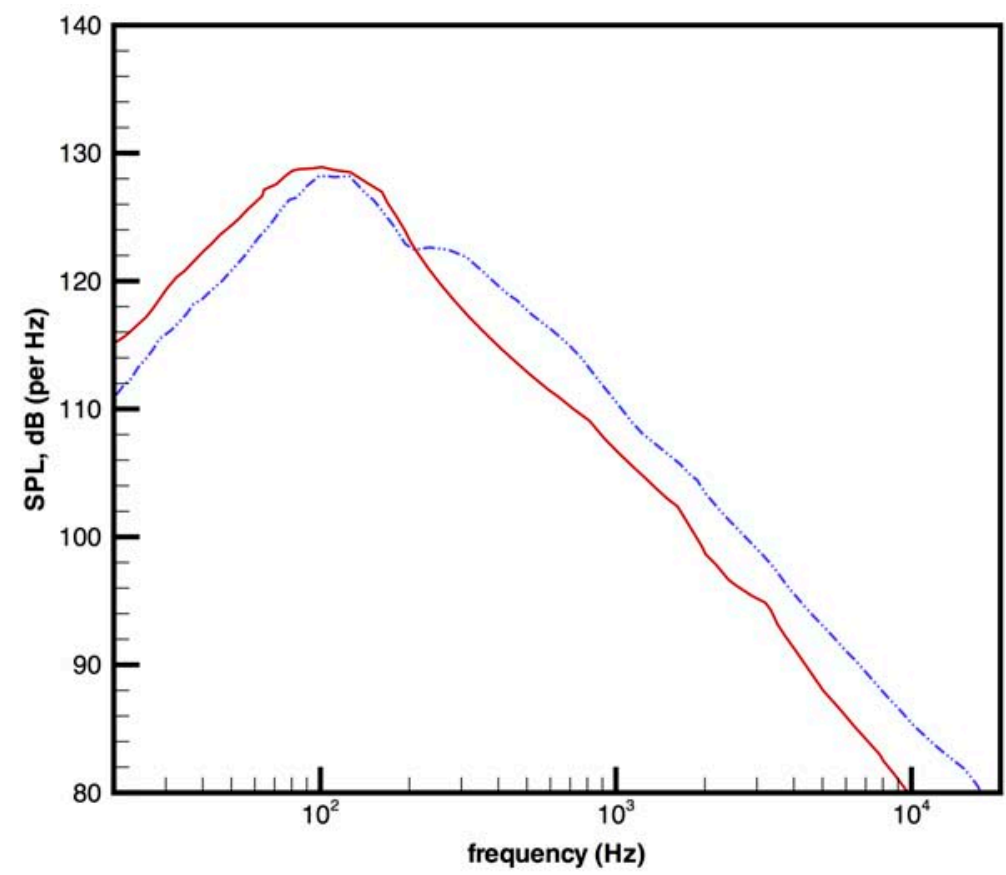

Figure 19. A superposition of the measured spectra at $130^{\circ}$ and $140^{\circ}$ directions. 


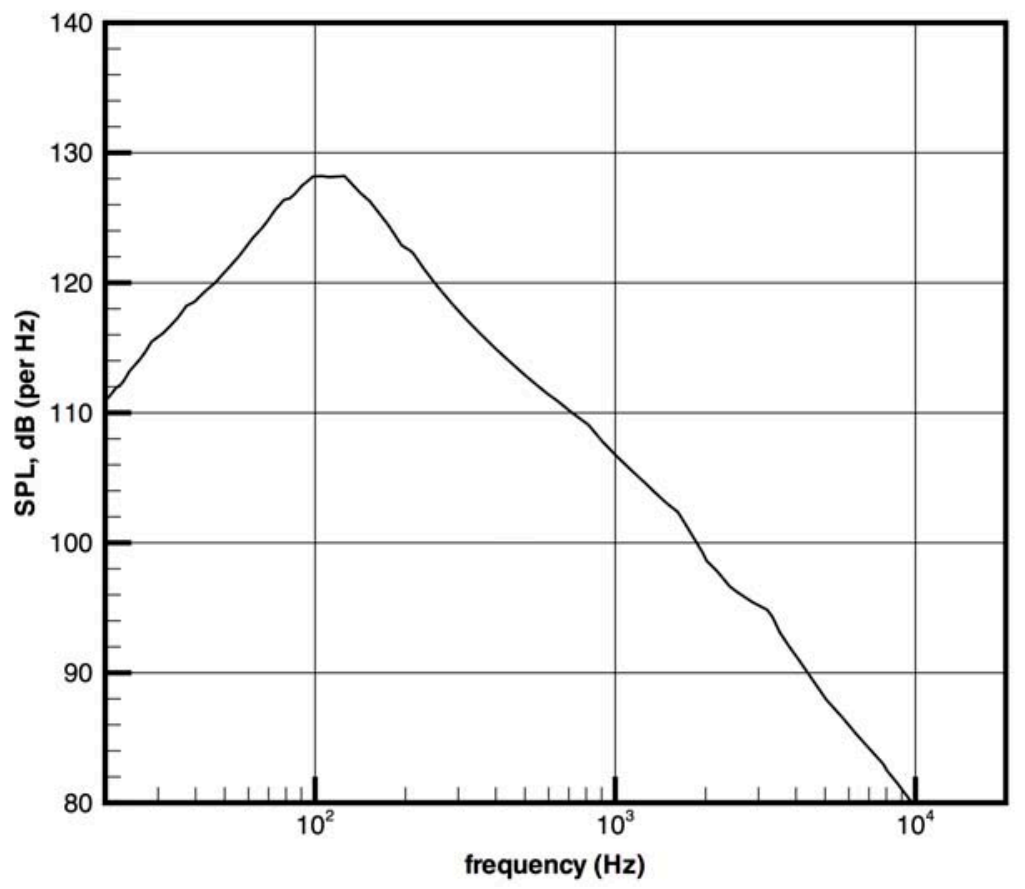

Figure 20. The large turbulence structures noise spectrum of the F-22A jet at afterburner.

Figures 21 to 27 show the noise spectra at $5^{\circ}$ intervals for angles between $110^{\circ}$ and $140^{\circ}$. In each of these figures, the spectrum of the large turbulence structures noise is shown as a dotted curve. Clearly, in the directions from $110^{\circ}$ to $130^{\circ}$, there is a new noise component in the spectrum. The new noise component appears to attain maximum intensity at around the $115^{\circ}$ direction. (Note: noise spectra in directions ending in $5^{\circ}$ are measured at a distance of $61 \mathrm{~m}$ from the nozzle exit. The data are from Gee et al [2]). One characteristic feature of this new noise component is that its spectral width reduces steadily, starting from $110^{\circ}$, as the inlet angle increases. This seems to suggest that there is a gradual cut-off of this noise component. The cutoff is most probably due to mean flow refraction. The cut-off starts with the high-frequency part of the spectrum, and the reduction process continues as inlet angle increases. By $140^{\circ}$, the new noise component is restricted to frequencies below those of the large turbulence structures noise. But its intensity is lower, so it is buried under the large turbulence structures noise spectrum and cannot be seen. 


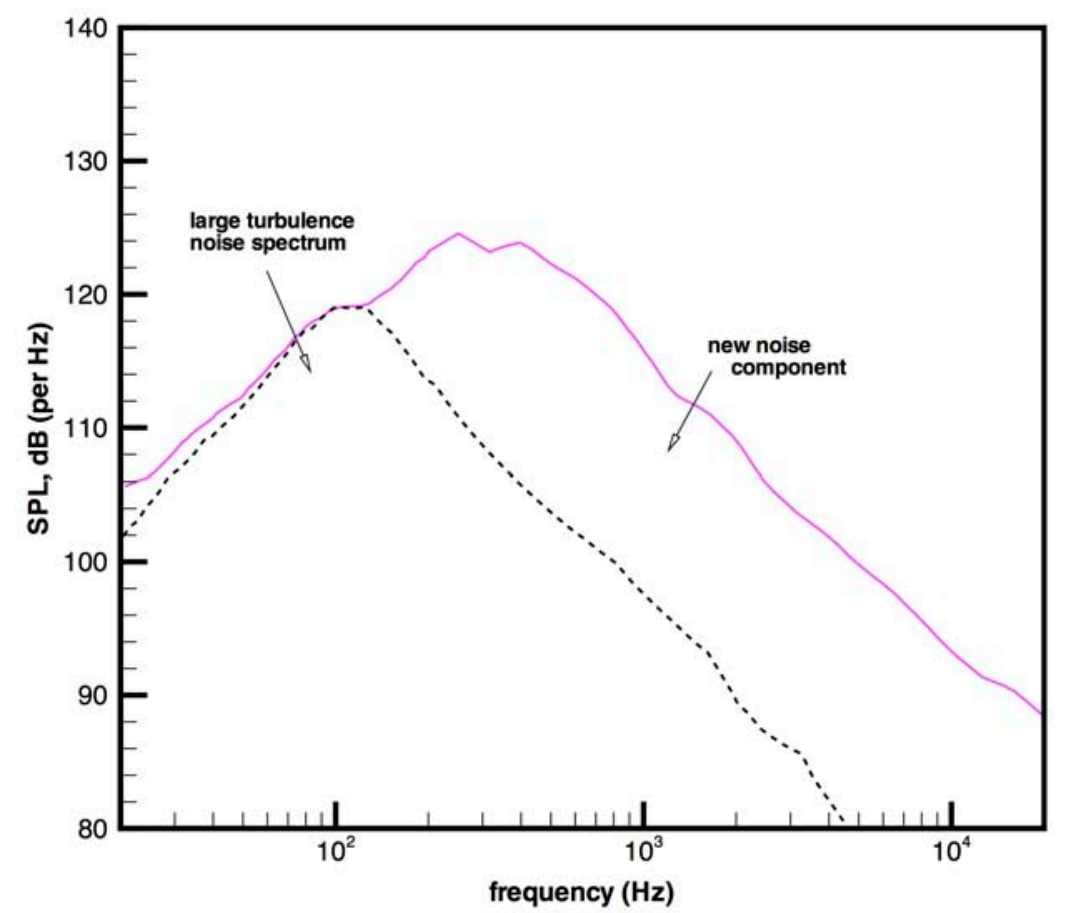

Figure 21. Measured noise spectrum in the $110^{\circ}$ direction, showing a new noise component. Dotted curve is the large turbulence structures noise spectrum.

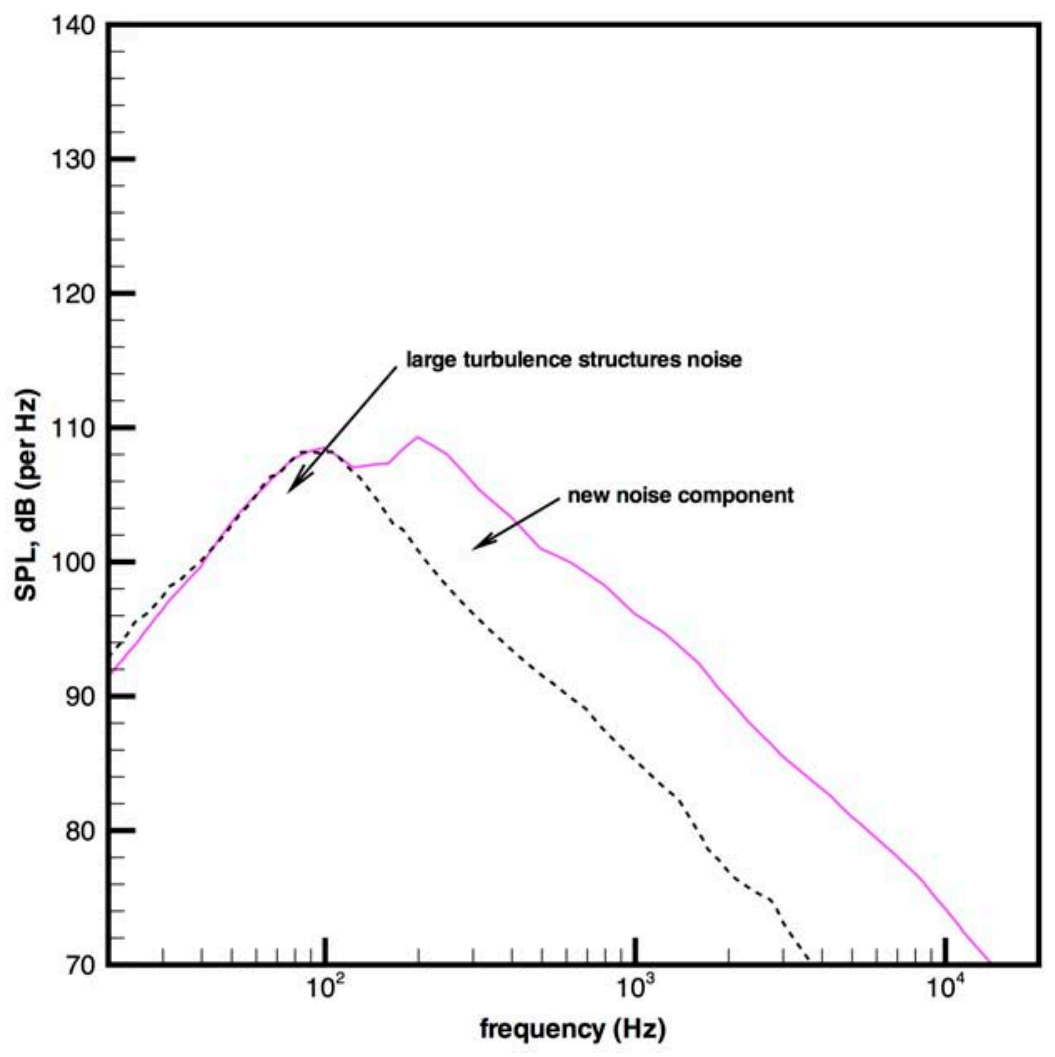

Figure 22. Measured noise spectrum in the $115^{\circ}$ direction, showing a new noise component. Dotted curve is the large turbulence structures noise spectrum. 


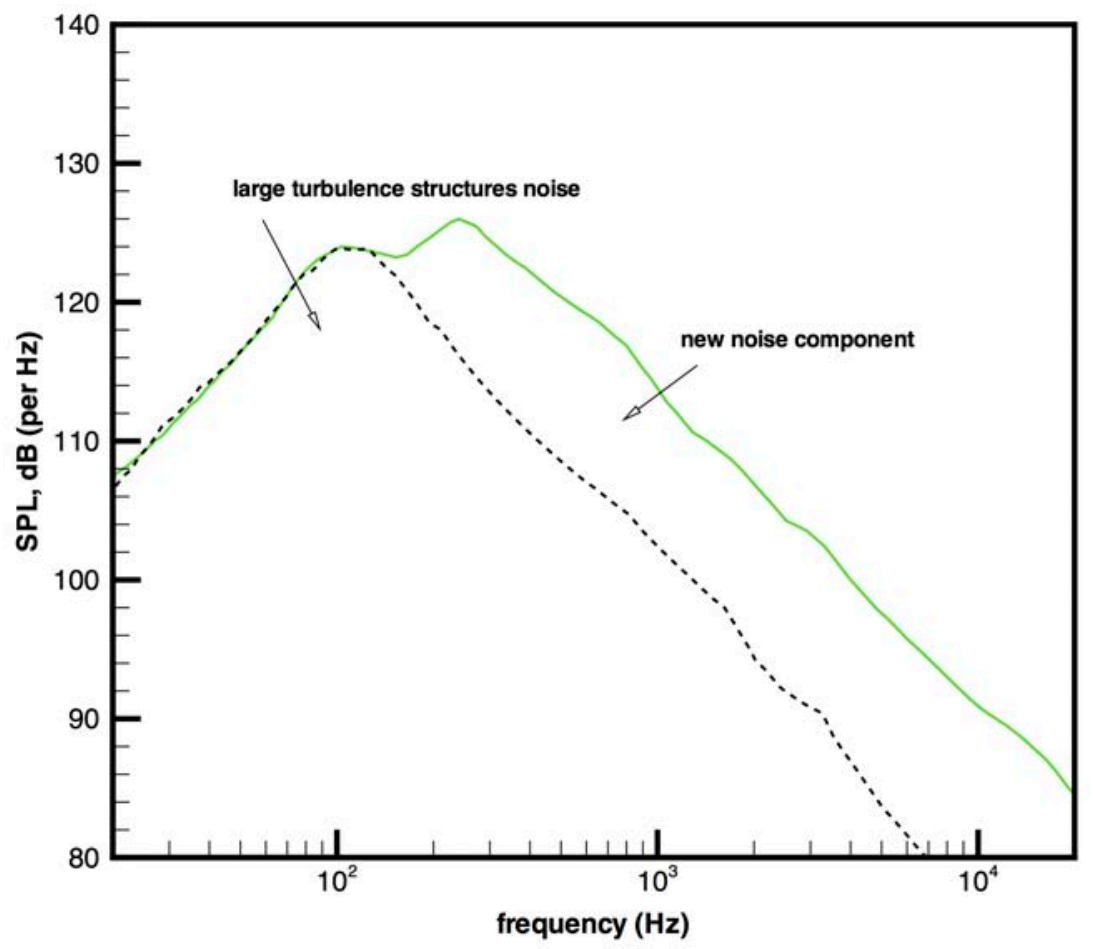

Figure 23. Measured noise spectrum in the $120^{\circ}$ direction, showing a new noise component. Dotted curve is the large turbulence structures noise spectrum.

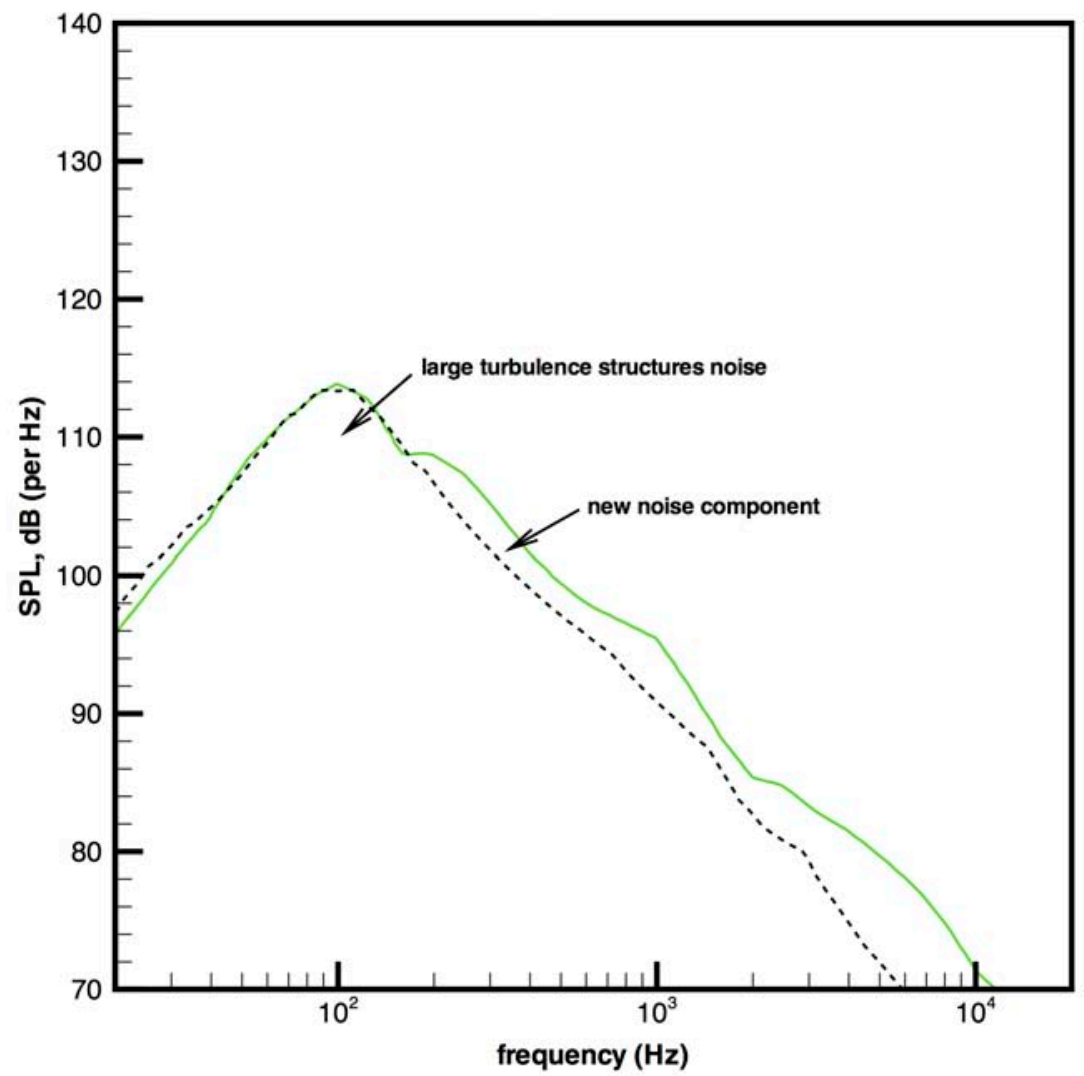


Figure 24. Measured noise spectrum in the $125^{\circ}$ direction, showing a new noise component. Dotted curve is the large turbulence structures noise spectrum.

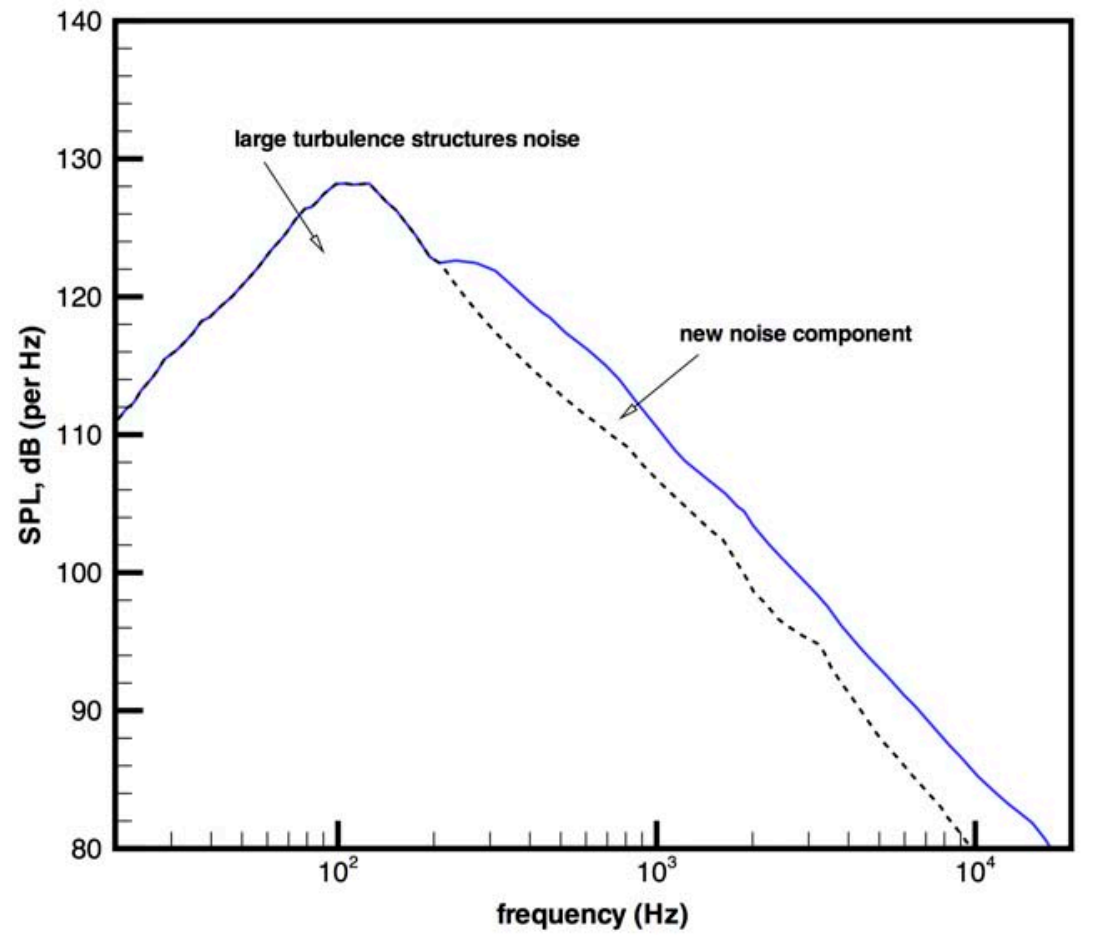

Figure 25. Measured noise spectrum in the $130^{\circ}$ direction, showing a new noise component. Dotted curve is the large turbulence noise spectrum.

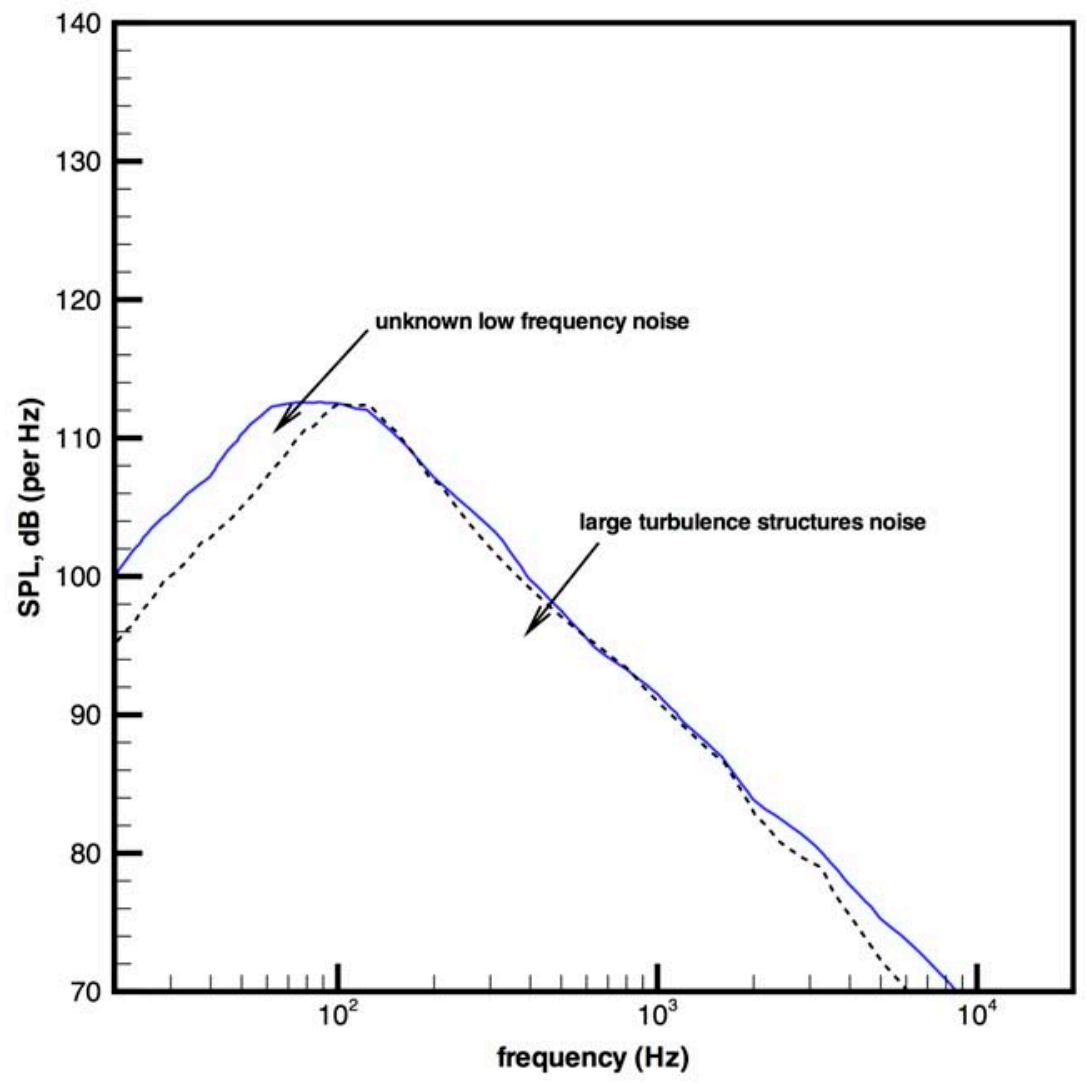


Figure 26. Measured noise spectrum in the $135^{\circ}$ direction, showing a new noise component. Dotted curve is the large turbulence structures noise spectrum.

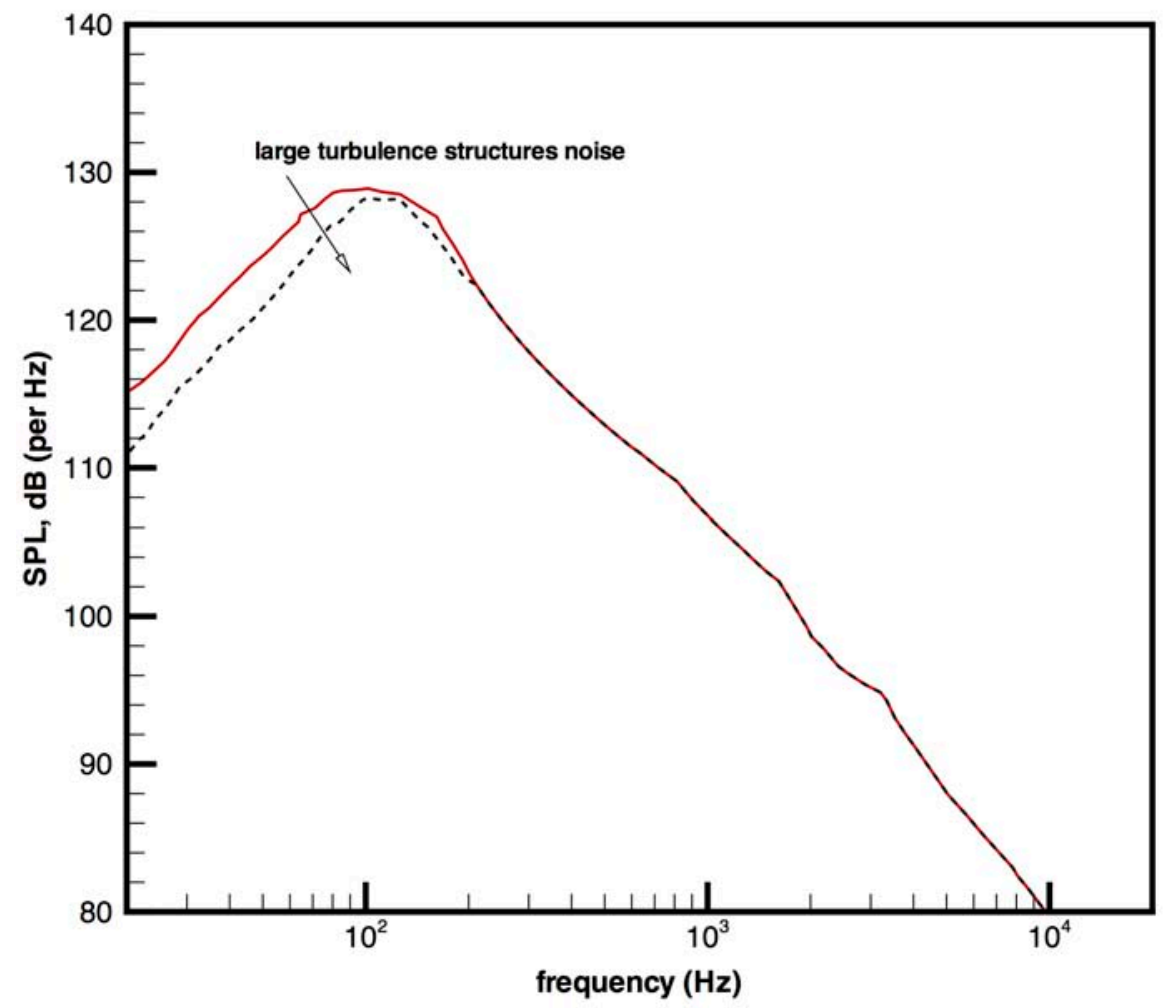

Figure 27. Measured noise spectrum in the $140^{\circ}$ direction. Dotted curve is the large turbulence structures noise spectrum.

Figures 28 and 29 show the noise spectra measured in the $145^{\circ}$ and $150^{\circ}$ directions. The noise spectrum of the large turbulence structures of the jet flow is plotted as the dotted curves in these figures. The entire spectrum consists of two components. The main component is the large turbulence structures noise. At very low frequencies, below $100 \mathrm{~Hz}$, there is an additional component. This component will be considered an unknown component for now. This unknown noise component appears to show up first in figure 26, the noise spectrum in the $135^{\circ}$ direction. It spreads to lower frequencies as the angle of radiation increases. The possible origin of this noise component will be discussed in a later section. 


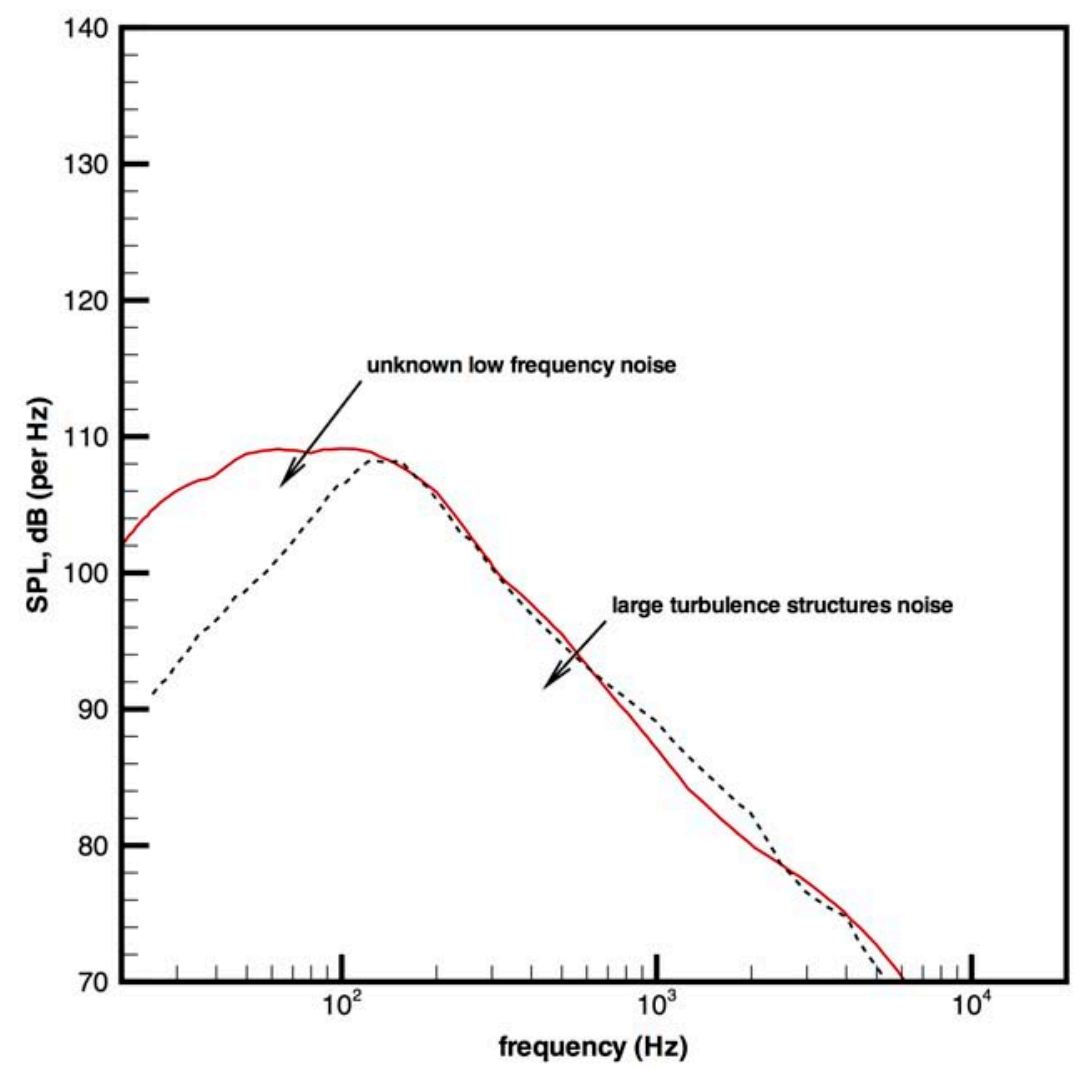

Figure 28. Measured noise spectrum in the $145^{\circ}$ direction. There is an unknown noise component at low frequencies. Dotted curve is the large turbulence structures noise spectrum.

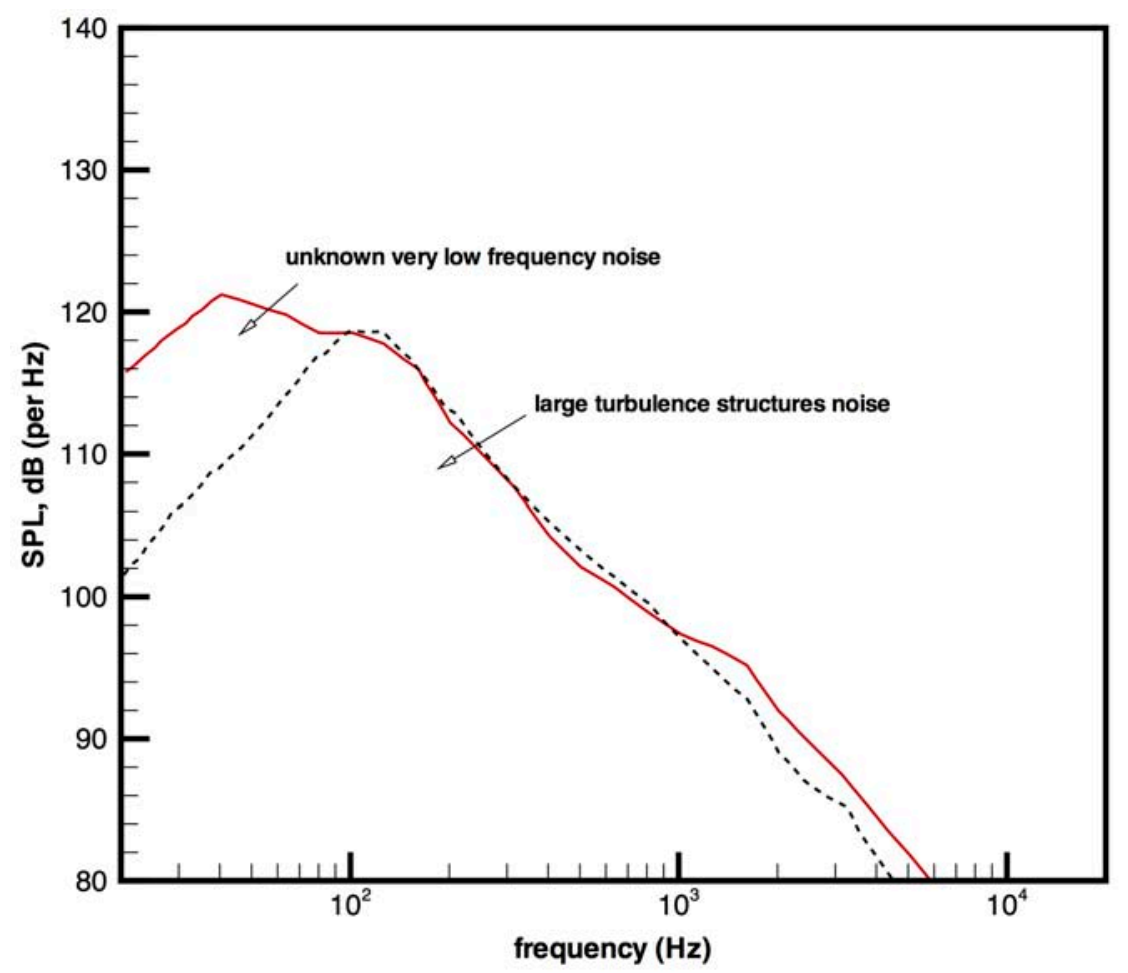

Figure 29. Measured noise spectrum in the $150^{\circ}$ direction. There is an unknown noise component at low frequencies. Dotted curve is the large turbulence structures noise spectrum. 
Figure 30 illustrates the two most dominant components of high-performance aircraft noise at afterburner. They are the low-frequency large turbulence structures noise and the new noise component at higher frequency. This figure includes noise spectra measured from $110^{\circ}$ to $150^{\circ}$ inlet angle at $10^{\circ}$ intervals. It shows that at $110^{\circ}$, the level of the large turbulence structures noise is lower than that of the new noise component. But as the inlet angle increases, the SPL of the large turbulence structures noise increases rapidly. This noise component attains its maximum intensity at around $140^{\circ}$. Its level drops off by $10 \mathrm{~dB}$ when the inlet angle increases further to $150^{\circ}$. On the other hand, the new noise component increases in intensity starting from approximately $100^{\circ}$. Its intensity peaks around $115^{\circ}$ and decays rapidly as the inlet angle increases further. From this figure, it is easy to see that the dominance of the two noise components switches hands at approximately $200 \mathrm{~Hz}$, with the new noise component more dominant at higher frequencies.

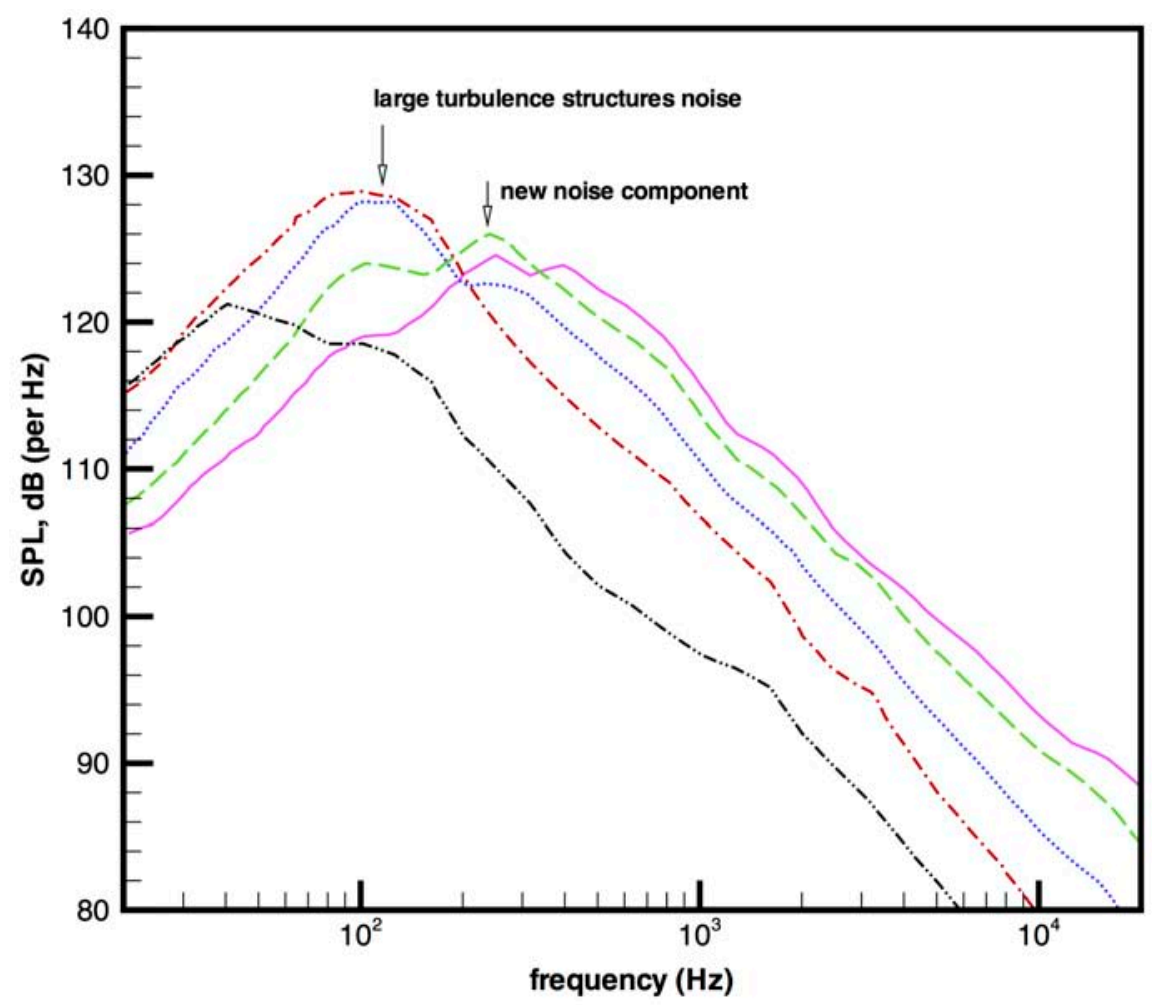

Figure 30. Noise spectra at various directions of radiation. Indicated by arrows in this figure are the two most dominant noise components. The dominance switches hands around $200 \mathrm{~Hz}$. $-110^{\circ},----120^{\circ}, \cdots \cdots 130^{\circ},-\cdot-140^{\circ},-\cdots-150^{\circ}$.

A different way of showing there are, indeed, two dominant noise components is to look at the directivities of noise contours at a fixed frequency. Figure 31 is a plot of the directivities from $300 \mathrm{~Hz}$ to $4000 \mathrm{~Hz}$. The direction of maximum noise radiation is around $115^{\circ}$. Figure 32 is a similar directivity plot for frequencies of 50,70 and $100 \mathrm{~Hz}$. For these low frequencies, the direction of maximum noise radiation is around $140^{\circ}$. Figure 33 is a combined plot of figures 31 and 32. It shows that for the F-22A aircraft, there are two distinct jet noise radiation patterns. This indicates that there are two distinctly different sources of noise (note: the $200 \mathrm{~Hz}$ contour exhibits two separate peak directions of radiation). The low-frequency component is the large turbulence structures noise. The high-frequency component comes from the new noise source. 


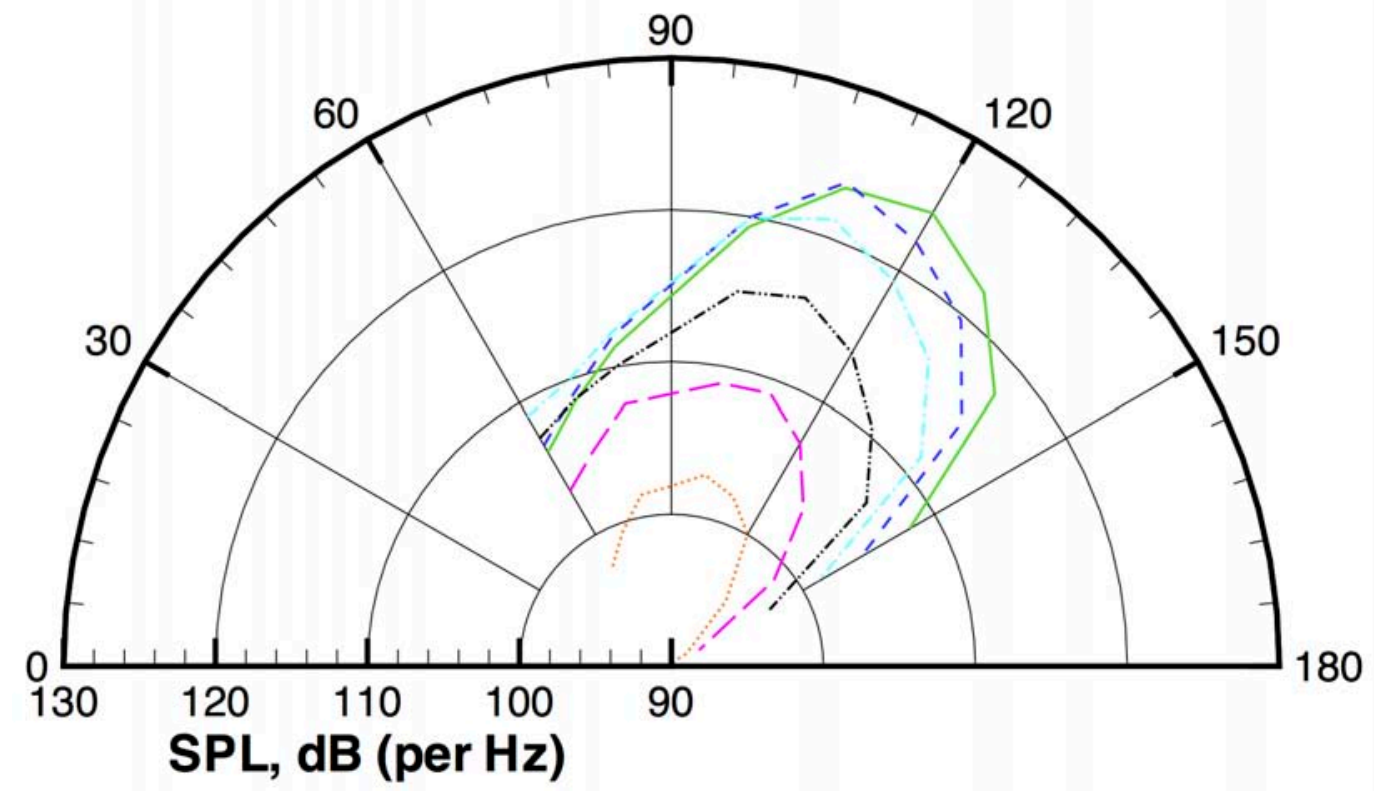

Figure 31. Directivities of pressure contours at fixed frequencies. Shown are contours for frequencies between $300 \mathrm{~Hz}$ and $4000 \mathrm{~Hz} .-300 \mathrm{~Hz},---400 \mathrm{~Hz},-\cdot-800 \mathrm{~Hz}$, $-\cdots-1000 \mathrm{~Hz},----2000 \mathrm{~Hz}, \cdots 4000 \mathrm{~Hz}$.

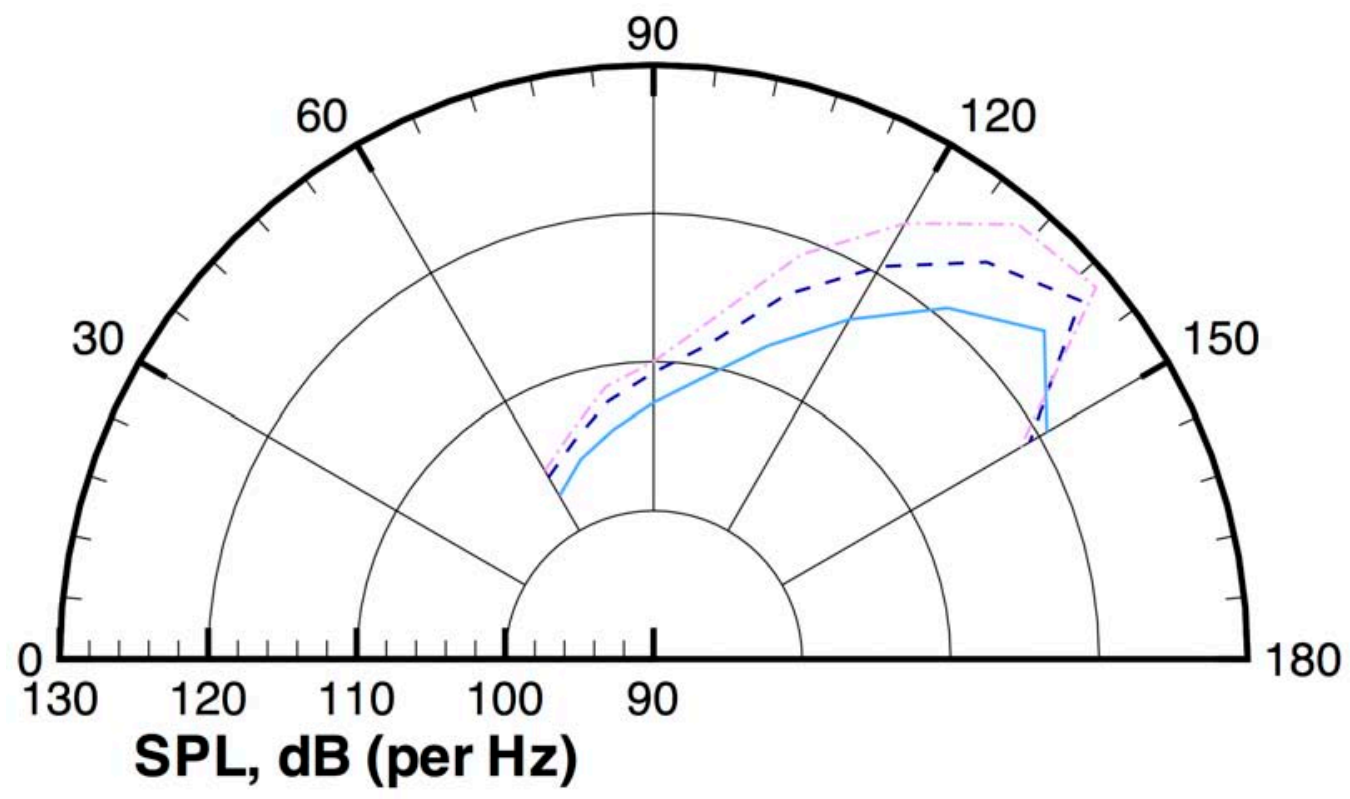

Figure 32. Directivities of pressure contours at fixed frequencies. Shown are contours for frequencies between $50 \mathrm{~Hz}$ and $100 \mathrm{~Hz} .-50 \mathrm{~Hz},---70 \mathrm{~Hz},-\cdot-100 \mathrm{~Hz}$. 


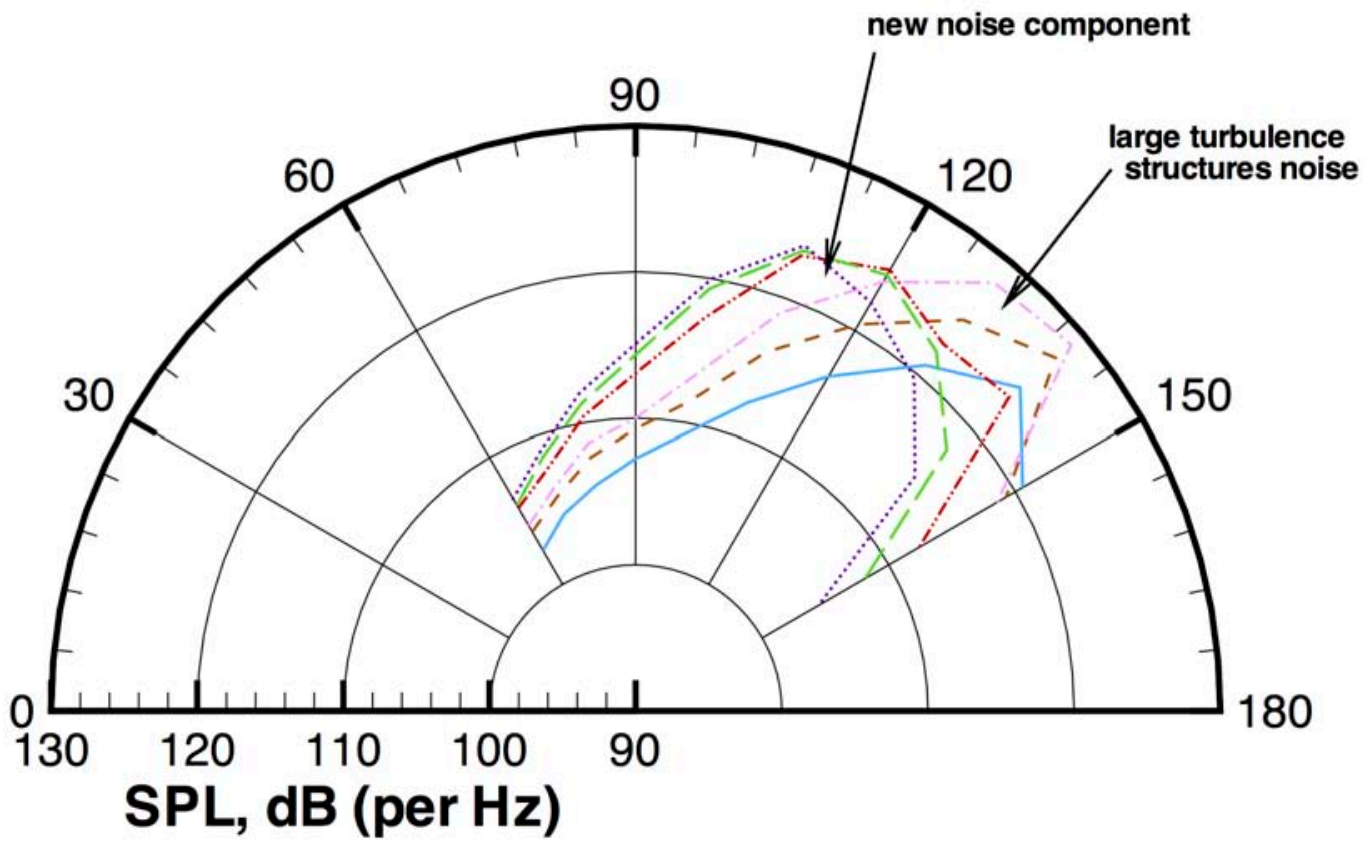

Figure 33. Directivities of pressure contours at fixed frequencies from $50 \mathrm{~Hz}$ to $400 \mathrm{~Hz}$. $-50 \mathrm{~Hz},---, 70 \mathrm{~Hz},-\cdot-100 \mathrm{~Hz},-\cdots-200 \mathrm{~Hz},---300 \mathrm{~Hz}, \cdots \cdots 400 \mathrm{~Hz}$.

To conclude the present data analysis, we would like to report that the noise of a highperformance aircraft at afterburner is a good deal more complex than that of a hot supersonic laboratory jet. In addition to the two components of turbulent mixing noise, there are two new noise components. Figure 34 is a schematic diagram showing the principal directions of radiation of these four noise components based on the analysis above. Overall, the noise of the large turbulence structures and the new noise component are the most dominant. Both components have nearly equal maximum noise intensity. The new noise component is dominant in the directions from about $100^{\circ}$ to $130^{\circ}$. Its intensity peaks around $115^{\circ}$. The large turbulence structures noise, on the other hand, is dominant in the directions of $110^{\circ}$ to $150^{\circ}$, peaking around $135^{\circ}$ to $140^{\circ}$. Other than these two most dominant noise components, the fine-scale turbulence noise is important for radiation in the upstream direction. In the downstream direction at inlet angles $135^{\circ}$ and larger, there is a very low-frequency unknown noise component. Because the available data covers only directions up to $150^{\circ}$, we are unable to offer further information on this unknown noise component at angles closer to the jet flow direction. 


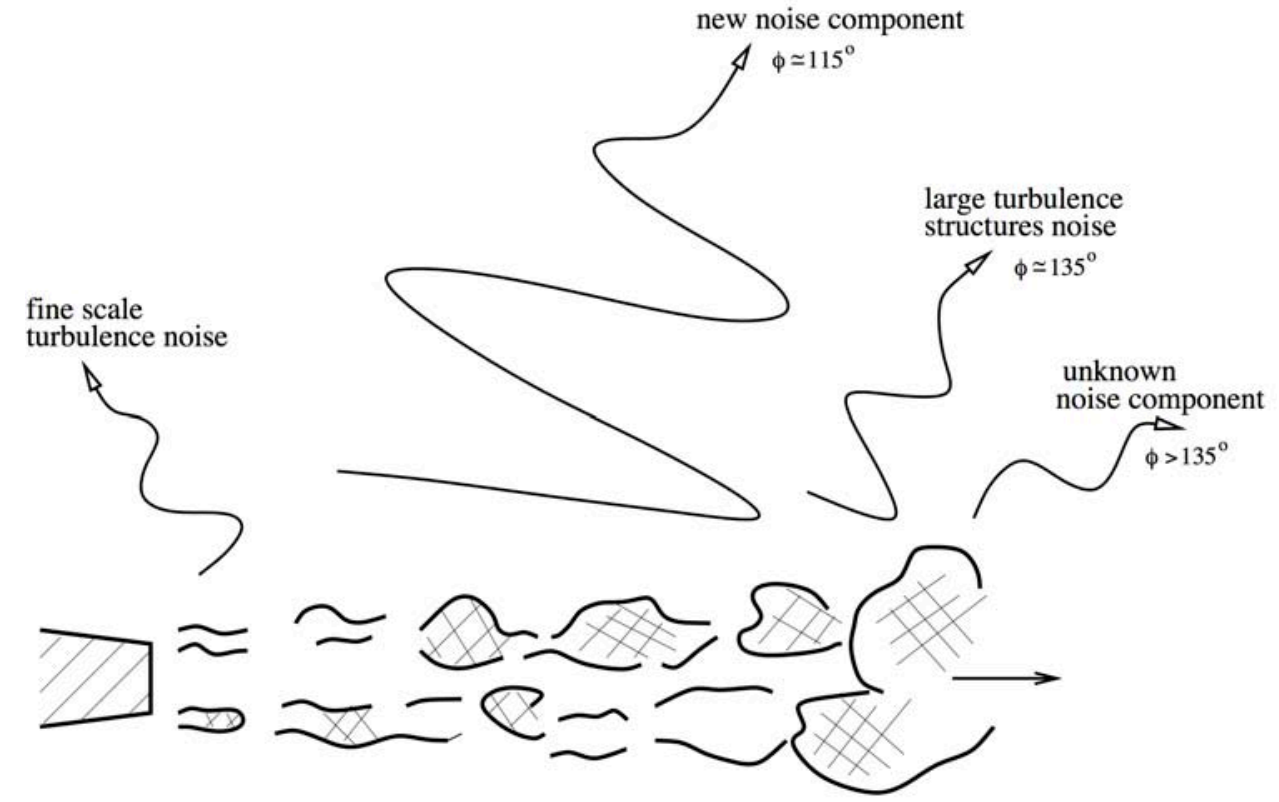

Figure 34. Schematic diagram of the directions of radiation for the four noise components identified from the F-22A noise data at afterburner. The large turbulence structures noise and the new noise component are the most dominant.

\section{The New Noise Component}

The analysis from the previous section leads to an important question: What is the source mechanism responsible for generating the new noise component? It is worthwhile to note that the new noise component emerges when the rate of fuel burn increases significantly above that of the intermediate power setting. This strongly suggests that the source mechanism is combustion related.

It is now known that there are two types of combustion noise: direct combustion noise and indirect combustion noise. When fuel is burned in a combustor, the chemical reaction generates both sound and hot spots. The sound generated is referred to as direct combustion noise. The hot spots or entropy waves are temperature and density fluctuations. They have no accompanying pressure fluctuations. However, when these hot spots/entropy waves are convected by the mean flow through a constriction in the engine ducting system, such as a turbine stage, or through a region with non-uniform mean flow, pressure fluctuations are generated. These pressure fluctuations immediately lead to sound radiation. This form of acoustic radiation is referred to as indirect combustion noise.

Mahan and Karchmer [15] in their review on combustion noise mentioned that direct combustion noise from open flames has a spectrum very much similar to that of a turbulent jet. Tam, Pastouchenko, Mendoza \& Brown [16] in their investigation of direct combustion noise from auxiliary power units (APU) found that the noise spectrum was identical to that of the large turbulence structures noise of high-speed jets (see figure 2). This being the case, it seems unlikely that the new noise component is direct combustion noise because we have already found in the previous section that the spectrum shape of the new noise component is unlike that of the similarity spectrum of the large turbulence structures noise. This leads us to believe that the new 
noise component of high-performance aircrafts at afterburner is most likely indirect combustion noise.

\subsection{Indirect combustion noise: a brief review}

Chu and Kovasznay [17] have shown that a uniform, compressible flow can support three types of independent small-amplitude disturbances. They referred to them as acoustic, vorticity and entropy waves. Entropy waves involve fluctuations in gas temperature and density only. There are no pressure or velocity fluctuations associated with this type of disturbance. Candel [18] and Marble and Candel [19] were among the first to recognize that when entropy waves were convected into a nonuniform region, as might be created by a flow constriction, these waves would couple to acoustic waves, leading to pressure fluctuations and acoustic radiation. In the 1970s and 1980s, research on indirect combustion noise was carried out by Morfey [20], FfowcsWilliams and Howe [21] Cumpsty [22], Cumpsty and Marble [23], Pickett [24] and Sinai [25]. But these studies were entirely theoretical. Experimental confirmation of the indirect combustion noise phenomenon was unsuccessful for a long period of time. Nearly forty years after these early studies, the existence of indirect combustion noise was finally confirmed experimentally by Bake, Kings, Fischer and Rohle [26]. Bake, Richter, Muhlbauer, Kings, Rohle, Thiele and Noll [27]. They designed and built a special facility known as the Entropy Wave Generator (EWG) for their breakthrough experiment. By means of their EWG, Bake et al. were able to obtain concrete evidence of the generation and radiation of indirect combustion noise.

Since the experimental confirmation of the existence of indirect combustion noise, there has been a renewed interest in the subject. Recently, a large number of papers on this subject were published, e.g., Leyko, Nicoud and Poinsot [28], Howe [29], Goh and Morgans [30, 31], Giauque, Huet and Clero [32], Duran, Moreau and Poinsot [33], Mishra and Bodony [34], Morgans, Goh and Dahan [35] and Tam and Parrish [36]. Because high temperature is involved, experimental investigation of indirect combustion noise remains a significant challenge. With rapid advances in computational methods in recent years, there is a growing interest in the use of numerical simulation to predict and to unravel the physics and generation mechanism of indirect combustion noise.

\subsection{Indirect combustion noise from a military-style nozzle}

To support the proposition that the new noise component is the result of indirect combustion noise, we would first like to demonstrate by numerical simulation that the passage of hot and cold spots through a military-style nozzle (see figure 35) would lead to the generation and radiation of indirect combustion noise. This is the best we can do for now as no experimentally measured flow and temperature data of the F-22A jets have been published. Since the geometry and operating conditions of military engines are classified information, we will use a model configuration, a static temperature, and a static pressure inside the afterburner, based on the best information available. To simplify the problem, we will assume that the nozzle flow is quasi-onedimensional. The size and spectrum of hot spots/entropy waves coming out of the afterburner of an F-22 aircraft are unknown and might not have even been measured. For this reason, we will consider two simulations. The first simulation has a single hot spot or an entropy wave pulse entering the upstream end of the computational domain as input. This simulation allows us to demonstrate that the indirect combustion noise generated consists both fast and slow acoustic waves. The second simulation uses a temperature wave train at a specified frequency as input. These simulations explore the effects of unsteady entropy wave input on indirect combustion noise generation. 


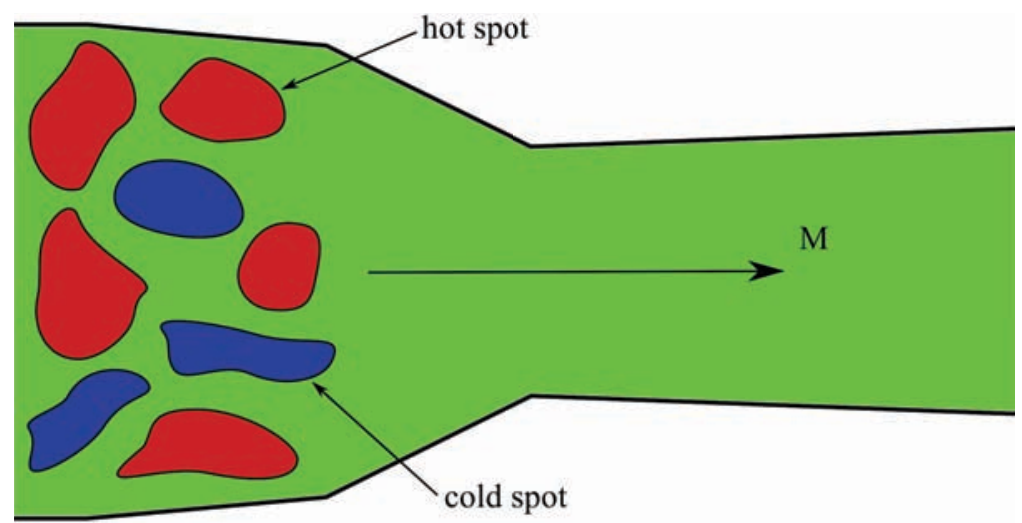

Figure 35. Schematic diagram of the generation of indirect combustion noise by the passage of hot and cold spots/entropy waves through a military-style nozzle.

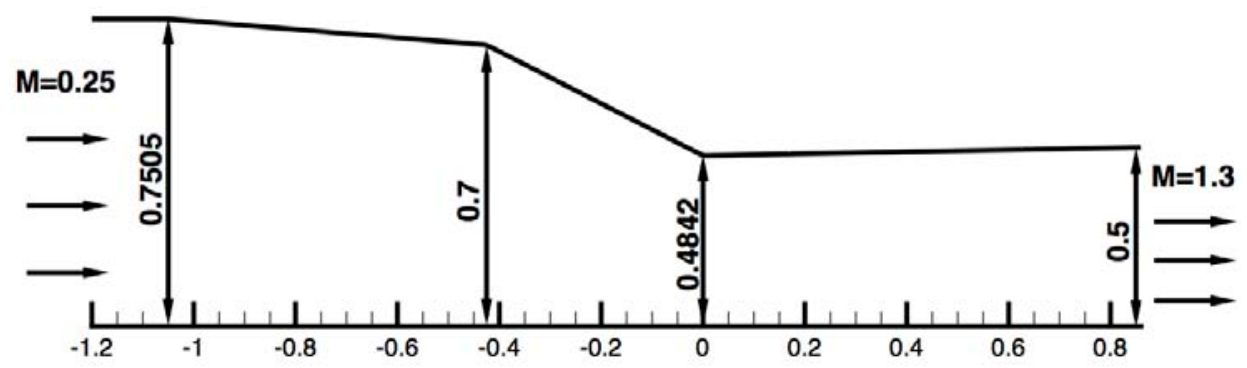

Figure 36. Geometry of the military-style nozzle and Mach numbers used for numerical simulation.

Figure 36 shows the nozzle geometry used in the present investigation. For convenience, the throat of the nozzle is taken as the origin of coordinates, i.e., $x=0$. The nozzle exit diameter is used as the length scale in the computation. The flow variables in the uniform flow region just upstream of the nozzle (the afterburner) will be used as reference variables. The duct radius upstream of $x=-1.2$ is taken to be constant. These variables are denoted by a subscript ' $r$ '. The following scales are used to form dimensionless variables in the simulations.

Length scale $=D$ (diameter at nozzle exit)

Velocity scale $=a_{r}$ (speed of sound in the reference station)

Time scale $=D / a_{r}$

Density scale $=\rho_{r}$

Pressure scale $=\rho_{r} a_{r}^{2}$

Temperature scale $=T_{r}$

The governing equations are the equations of conservation of mass, momentum and thermal energy. In terms of variables $T, p, u$ and nozzle area $A(x)$, they are, 


$$
\begin{aligned}
& \frac{\partial T}{\partial t}+u \frac{\partial T}{\partial x}+(\gamma-1) T \frac{\partial u}{\partial x}+(\gamma-1) \frac{u T}{A} \frac{d A}{d x}=0 \\
& \frac{\partial u}{\partial t}+u \frac{\partial u}{\partial x}+\frac{T}{\gamma p} \frac{\partial p}{\partial x}=0 \\
& \frac{\partial p}{\partial t}+u \frac{\partial p}{\partial x}+\gamma p \frac{\partial u}{\partial x}+\gamma \frac{p u d A}{A} \frac{d x}{d x}=0
\end{aligned}
$$

The computational domain extends from $x=-1.2$ to $x=1.0$, with the variable-area part of the nozzle between $x=-1.05$ and $x=0.85$, as in the nozzle of Fig. 36. The mean flow and the entropy wave pulse (in the form of a Gaussian with half-width equal to 3 mesh spacings) enter the computational domain from the left boundary. Exiting from this boundary are upstream propagating indirect combustion acoustic waves generated inside the nozzle. The mean flow, the entropy wave pulse and the downstream propagating indirect combustion noise all exit the computational domain at the right boundary. To ensure there is little reflection of the flow disturbances back into the computational domain, a perfectly matched absorbing layer (PML) (see Hu [37], [38]) is installed at the left boundary. At the right boundary, no special treatment is required, as the mean flow is supersonic. Before carrying out the time-dependent numerical simulation, the mean flow is first computed by time marching the discretized governing equations of (2), (3) and (4) to a time-independent state. There is an analytical mean flow solution to the governing equations. This analytical solution is used as the starting solution for the mean flow computation. The incoming and outgoing entropy wave pulse and the indirect combustion noise generated inside the nozzle are relatively small in amplitude. Therefore, a linearized treatment of these disturbances at the upstream boundary region is quite acceptable. The linearized PML equations with respect to a uniform mean flow, $\bar{u}$, pressure, $\bar{p}$, and temperature, $\bar{T}$, are,

$$
\begin{aligned}
& \frac{\partial T^{\prime}}{\partial t}+\sigma(1+\beta \bar{u}) T^{\prime}+\sigma \beta(\gamma-1) \bar{T} u^{\prime}+\bar{u} \frac{\partial T^{\prime}}{\partial x}+(\gamma-1) \bar{T} \frac{\partial u^{\prime}}{\partial x}=0 \\
& \frac{\partial u^{\prime}}{\partial t}+\sigma(1+\beta \bar{u}) u^{\prime}+\sigma \beta \frac{\bar{T}}{\gamma \bar{p}} p^{\prime}+\bar{u} \frac{\partial u^{\prime}}{\partial x}+\frac{\bar{T}}{\gamma \bar{p}} \frac{\partial p^{\prime}}{\partial x}=0 \\
& \frac{\partial p^{\prime}}{\partial t}+\sigma(1+\beta \bar{u}) p^{\prime}+\sigma \beta \gamma \bar{p} u^{\prime}+\bar{u} \frac{\partial p^{\prime}}{\partial x}+\gamma \bar{p} \frac{\partial u^{\prime}}{\partial x}=0
\end{aligned}
$$

where $\beta=\frac{\bar{u}}{\bar{T}-\bar{u}^{2}}$ and $\sigma(x)$ is the damping function. At the left boundary region, there are incoming and outgoing disturbances. To handle these dual roles, the split-variable method (see chapter 9 of the book by Tam [39]) is implemented.

To carry out the indirect combustion noise simulation, the governing equations (equations (2), (3) and (4)) are discretized according to the dispersion-relation-preserving (DRP) scheme (Tam \& Webb [40], Tam [39]). For convenience, a uniform mesh size is used throughout the entire computational domain. The numerical mean flow solution on the chosen grid is first 
computed. Figure 37 shows the computed mean flow solution over the entire length of the nozzle. This mean flow is then used as the initial condition for the acoustic computation. In the acoustic computation the input disturbance is allowed to enter the computational domain from the left boundary region. The same discretized equations (2), (3) and (4) are used. The computation continues until the incoming entropy wave pulse has exited the computational domain. For the time-periodic temperature wave train input disturbance, the computation continues until the numerical solution is time periodic. To find the indirect combustion noise, the mean flow solution is subtracted from the computed solution with incoming disturbances.

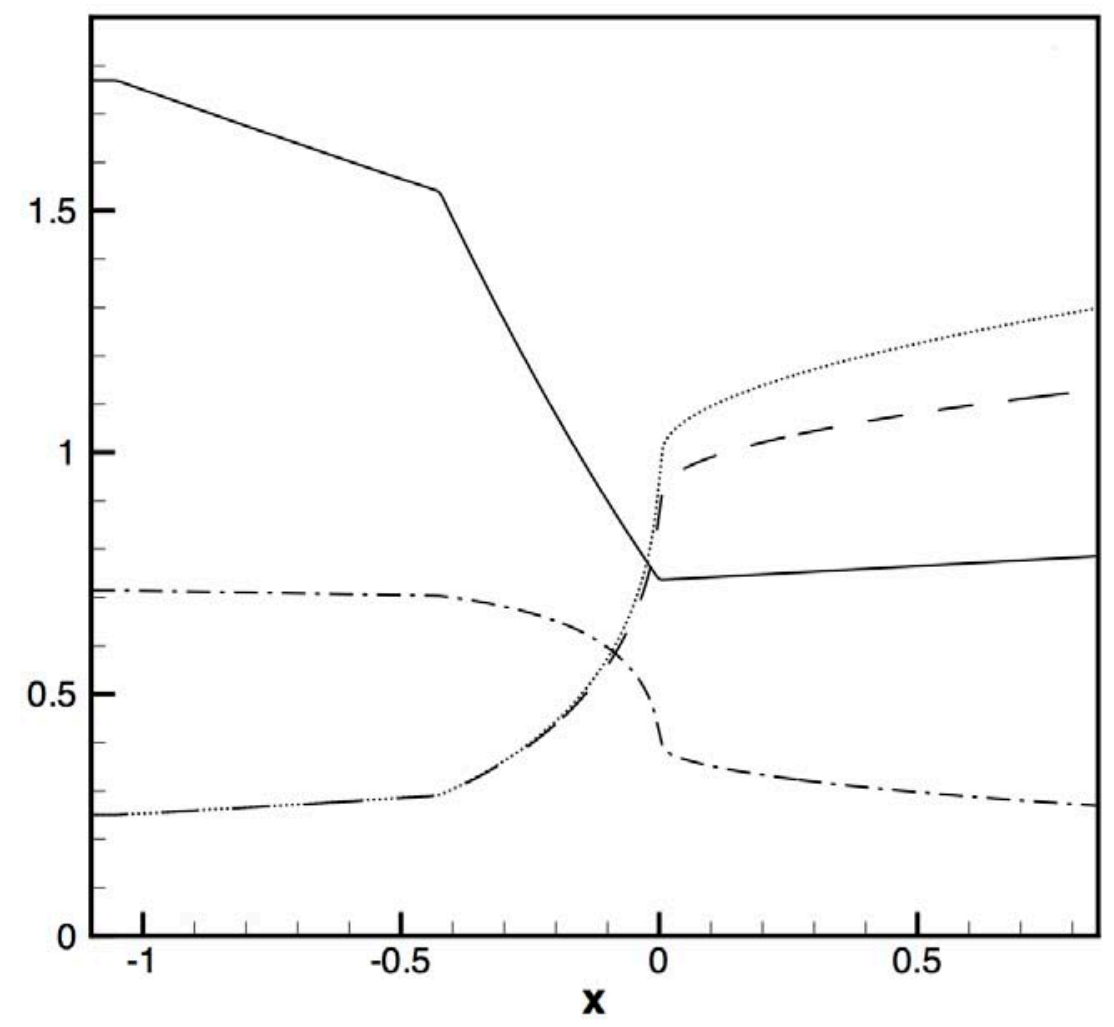

Figure 37. Computed mean flow quantities inside the nozzle of figure 36. Area of nozzle, $---\mathrm{u},-\cdot-\mathrm{p}, \cdots \cdots \mathrm{M}$.

\subsection{Results of numerical simulations}

For the numerical simulation of the passage of a Gaussian entropy wave pulse through a nozzle, the temperature distribution of the pulse just upstream of the computational domain is taken to be,

$$
T^{\prime}=A \exp \left\{-(\ln 2)\left[\frac{x-x_{u}-u_{u} t}{3 \Delta x}\right]^{2}\right\}, \quad p^{\prime}=u^{\prime}=0
$$


In equation (8), $x_{u}$ is the point located a distance of $3 \Delta x$ to the left of the upstream boundary of the computational domain and $u_{u}$ is the constant mean flow velocity in the boundary region. $\Delta x$ is the mesh size, and $A$ is the maximum pulse amplitude.
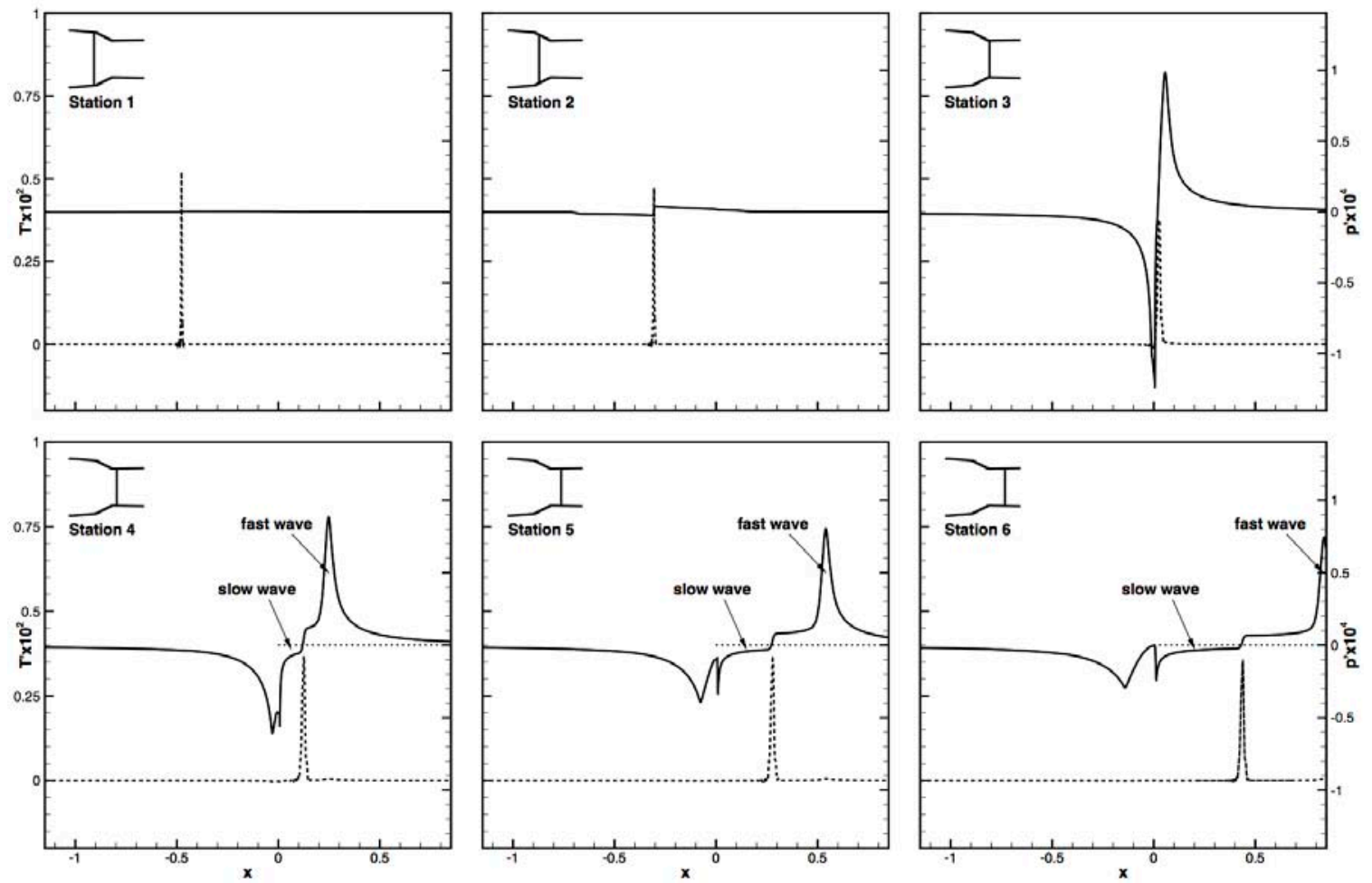

Figure 38. Instantaneous pressure distributions in a military-style convergent-divergent nozzle during the passage of an entropy wave pulse through the nozzle.

Soon after the numerical simulation has started, the entropy wave pulse enters the computational domain from the left boundary. Sample results of a simulation for the militarystyle nozzle of figure 36 are shown in figure 38. This figure shows the entropy wave pulse at six locations inside the nozzle. The location of the pulse is indicated in the inset at the upper left corner of each figure. The dotted line in the figure shows the temperature distribution (scale is on the left side of the figure); the solid line shows the pressure distribution (scale is on the right side of the figure). At station 1, the pulse, having an initial maximum amplitude of 0.01 , has just entered the variable area region of the nozzle. There is no pressure fluctuation. Station 2 is in the convergent part of the nozzle. Here, the mean flow velocity begins to accelerate. Now there is a pressure rise in the front part of the pulse. The physical mechanism that leads to the observed pressure rise is discussed in Tam \& Parrish [36]. The pressure rise immediately causes the radiation of a compression wave in the downstream direction. At the same time, it is easy to see that the pressure behind the pulse decreases, which results in the emission of a rarefaction wave in the upstream direction. Station 3 is positioned at the nozzle throat. For a military-style nozzle, the nozzle geometry changes abruptly from convergent to divergent at the throat. This creates a huge mean flow velocity gradient. As a result, when the entropy pulse reaches the nozzle throat, there is a large pressure increase in the front part of the pulse and an equally large pressure decrease at the back. This causes the emission of a large positive pressure pulse propagating ahead of the entropy pulse and a similarly large negative pressure pulse propagating away from the entropy pulse in the upstream direction. It is to be noted that the entropy wave pulse is 
convected downstream by the mean flow. Downstream of the nozzle throat, the mean flow is supersonic, so any acoustic waves generated on the backside of the pulse when the pulse is in the divergent part of the nozzle will not be able to propagate through the nozzle throat to the convergent part of the nozzle. Instead, these acoustic waves will be swept out of the nozzle downstream with a velocity between $\bar{u}$ (the local mean flow velocity) and $(\bar{u}-\bar{a})$, where $\bar{a}$ is the local speed of sound, by the supersonic mean flow. These are the slow waves, as they have a speed less than or equal to $\bar{u}$. On the downstream side of the entropy wave pulse, the compression waves have a speed ranging from $\bar{u}$ to $(\bar{u}+\bar{a})$. These are the fast waves and have a speed faster than that of the mean flow. The pressure distributions at stations 4,5 and 6 illustrate the creation of the fast and slow waves as the entropy wave pulse moves through the divergent part of the nozzle. In summary, the present numerical simulation shows that the passage of an entropy wave pulse through the convergent part a supersonic nozzle would cause the radiation of a compression wave downstream and a rarefaction wave upstream. In the divergent part of the nozzle, both waves propagate downstream. This gives rise to the emission of fast and slow waves, as illustrated schematically in figure 39.

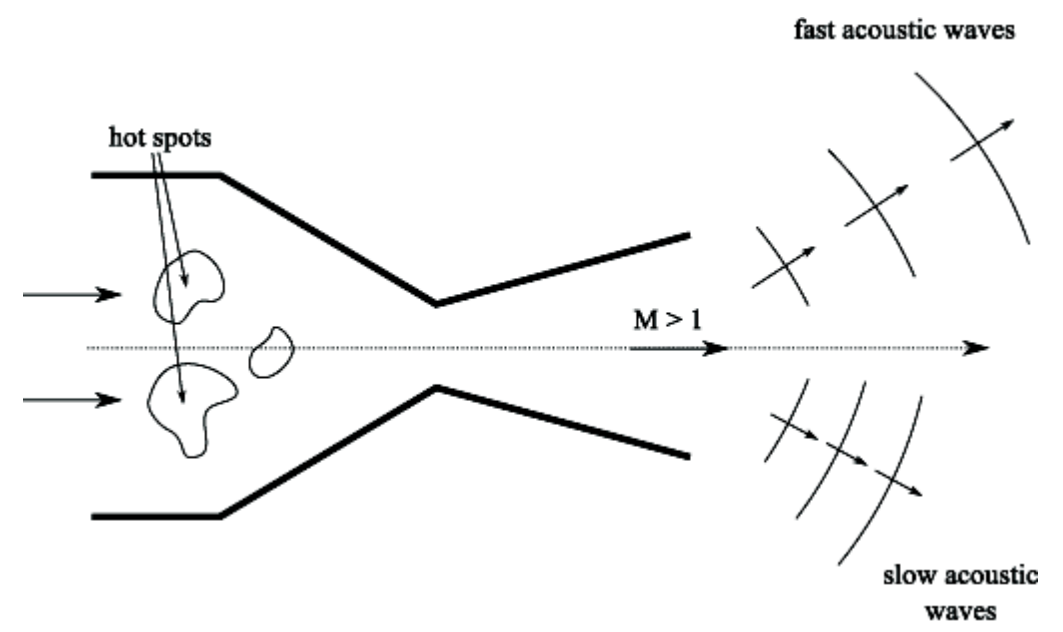

Figure 39. The passage of entropy waves/hot spots through a convergent-divergent supersonic nozzle leads to the emission of fast and slow acoustic waves from the nozzle exit.

What comes out of an afterburner, however, is not just a single hot spot. The situation is most likely resembling that as sketched in Fig. 35. One important feature of the entropy waves is the unsteady fluctuations in temperature. A simple way to model of an unsteady entropy wave is to use a time-periodic temperature wave train as incoming disturbances. Such a model simulation has been carried out. Figure 40 is a plot of the instantaneous temperature and pressure distributions inside the nozzle of figure 36. In this simulation, the diameter at the nozzle exit is taken to be $1.0 \mathrm{~m}$. The incoming temperature wave train has maximum dimensionless amplitude of $T^{\prime}=0.01$ at a frequency of $300 \mathrm{~Hz}$. The temperature distribution is shown as a dotted curve, with dimensionless temperature scale on the left side of the figure; the pressure distribution is shown as a solid line, with pressure scale on the right side of the figure. The nozzle configuration is also shown. This figure clearly reveals that the passage of a temperature wave train through the nozzle creates a large pressure fluctuation at the nozzle throat. This fluctuating pressure radiates sound waves upstream and downstream. The downstream propagating acoustic waves have a fairly complex waveform due to the superposition of fast and slow waves. In our simulations, the 
dimensionless quantity $\overline{p^{\prime 2}}$ (or, in dimensional form, $\frac{\overline{p^{\prime 2}}}{\rho_{r}^{2} a_{r}^{4}}$ ) is measured near the nozzle exit. In the next subsection, this quantity will be used to estimate the Intensity Level (IL) of the acoustic waves radiating out of a military-style nozzle due to the generation of indirect combustion noise inside the nozzle.

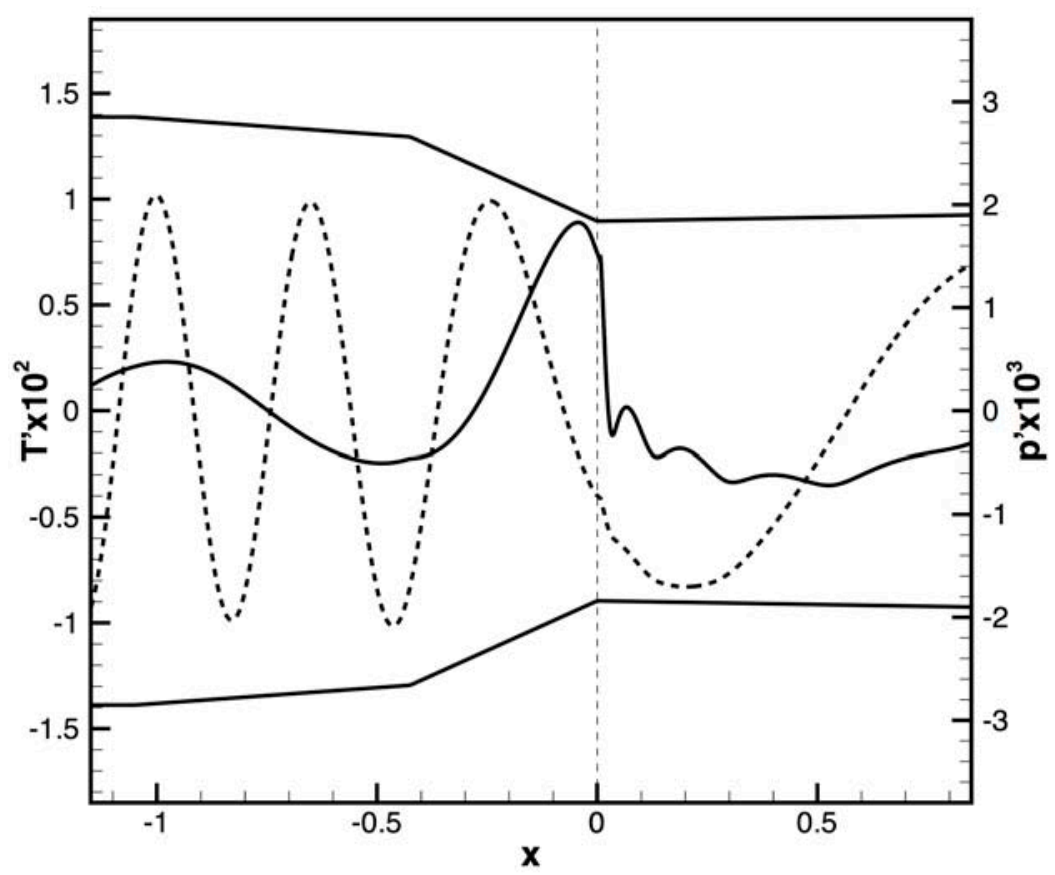

Figure 40. Indirect combustion noise generation due to the passage of a time-periodic entropy wave train through a military-style supersonic convergent-divergent nozzle. Shown in the figure are the instantaneous temperature (dotted curve; scale is on the left side of the figure) and pressure (full curve; scale is on the right side of the figure) distributions inside the nozzle.

\subsection{An estimate of noise directivity and intensity}

In this subsection, results of the quasi-one-dimensional simulations of indirect combustion noise generation from the previous subsection are used to estimate the directivity and intensity of the radiated noise. For this purpose, let us assume that the nozzle geometry is as shown in figure 36 and that it has a design Mach number of 1.3. The temperature inside the afterburner (just upstream of the nozzle) is taken to be $1200 \mathrm{~K}$ (speed of sound, $a_{r}=694 \mathrm{~m} / \mathrm{s}$ ). On assuming isentropic flow, the temperature, density and pressure relationship between the gas of the fully expanded jet and those inside the afterburner are given by,

$$
\frac{T_{r}}{T_{j}}=\frac{\left(1+\frac{\gamma-1}{2} M_{j}^{2}\right)}{\left(1+\frac{\gamma-1}{2} M_{r}^{2}\right)}, \quad \frac{\rho_{r}}{\rho_{j}}=\left[\frac{1+\frac{\gamma-1}{2} M_{j}^{2}}{1+\frac{\gamma-1}{2} M_{r}^{2}}\right]^{\frac{1}{-1}}, \quad \frac{p_{r}}{p_{j}}=\left[\frac{1+\frac{\gamma-1}{2} M_{j}^{2}}{1+\frac{\gamma-1}{2} M_{r}^{2}}\right]^{\frac{\gamma}{1-1}}
$$

where subscript ' $r$ ' denotes reference variables, i.e., variables inside the afterburner, and subscript ' $j$ ' denotes variables of the fully expanded jet flow. On using equation (9), the fully expanded jet flow variables are found to be $T_{j}=896 K, a_{j}=599 \mathrm{~m} / \mathrm{s}$ and $u_{j}=780 \mathrm{~m} / \mathrm{s}$. 
The acoustic waves radiated out of the nozzle consist of both fast and slow waves. Their wave speeds are,

$$
u_{j} \leq u_{\text {fast }} \leq\left(u_{j}+a_{j}\right), \quad\left(u_{j}-a_{j}\right) \leq u_{\text {slow }} \leq u_{j}
$$

The fast waves, the large turbulence structures of the jet flow and the slow waves all have velocity components faster than the ambient speed of sound $(340 \mathrm{~m} / \mathrm{s})$. Thus, they all are capable of generating Mach wave radiation, as illustrated in figure 5. For low supersonic jets, the large turbulence structures of the jet flow have a speed approximately equal to 0.7 of the jet speed (see Tam [5], Tam, Seiner \& Yu [41]). For an estimate of the dominant direction of radiation, we will use the Mach wave inlet angle, $\phi$, where $\phi=180^{\circ}-\hat{\phi}$ and $\hat{\phi}=\cos ^{-1}\left(a_{\infty} / u_{\text {source }}\right)$. On using a similar approximation for the fast waves, it is easy to find the directivity results shown in Table 1.

Table 1. Principal direction of radiation

\begin{tabular}{|c|c|c|}
\hline Source of noise & $u_{\text {source }} \mathrm{m} / \mathrm{s}$ & Principal direction of radiation, $\phi=180^{\circ}-\hat{\phi}$ \\
\hline $\begin{array}{c}\text { Large turbulence } \\
\text { structures }\end{array}$ & $0.7 u_{j}=546 \mathrm{~m} / \mathrm{s}$ & $129^{\circ}$ \\
\hline Fast waves & $0.7\left(u_{j}+a_{j}\right)=965 \mathrm{~m} / \mathrm{s}$ & $111^{0}$ \\
\hline Slow waves & Less than $0.7 u_{j}$ & $\phi_{\text {slow wave }}>135^{\circ}$ (see Fig. 25) \\
\hline
\end{tabular}

Figure 41 shows approximately the dominant direction of acoustic radiation of the large turbulence structures of the jet flow and those of the fast and slow waves. It is readily seen from Table 1 that the dominant direction of noise radiation for the large turbulence structures of the jet flow as computed is in good agreement with the summary of noise data shown in figure 34 . On treating the fast waves in a similar manner, the principal direction of radiation of the fast waves is found to be $111^{\circ}$. This is in close agreement with the principal direction of radiation of the new noise component in figure 34. Noise radiation from the slow waves will be in directions with inlet angle larger that of the large turbulence structures of the jet flow. This is similar to the radiation directions of the unknown noise component found in the previous section (see figure 34). Overall, although a very simple model is used in the directivity computation, the fact that there are reasonable agreements with measurements offers a fair degree of support to the proposition that the new and unknown noise components are, indeed, the results of indirect combustion noise generated inside the nozzle. 


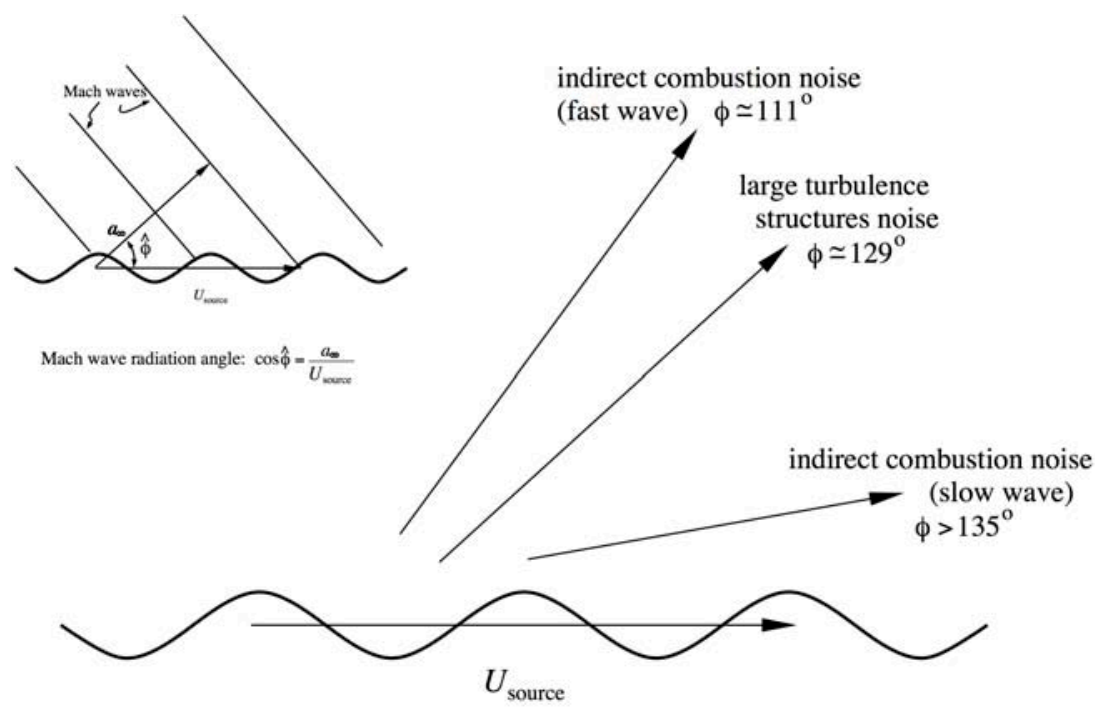

Figure 41. Estimated principal direction of radiation of various noise components (see Table 1).

It is of interest and relevance to estimate the noise intensity that the indirect combustion noise mechanism would generate in a Mach 1.3 nozzle. For this purpose, the acoustic energy flux formula developed by Cantrell and Hart [42] and Morfey [43] is used. We will use $\bar{I}$ to denote the energy flux from the nozzle, an overbar to denote a time-averaged quantity, and a prime to denote a time-fluctuating variable. For one-dimensional flow, the Cantrell and Hart formula may be rewritten as,

$$
\bar{I}=\bar{\rho} \bar{u} \overline{u^{2}}+\bar{u}^{2} \overline{\rho^{\prime} u^{\prime}}+\frac{\bar{u}}{\bar{\rho} \bar{a}^{2}} \overline{p^{\prime 2}}+\overline{p^{\prime} u^{\prime}}
$$

Additionally, if the flow occurs in a nozzle with a nearly constant area at its exit, formula (10) further simplifies to,

$$
\bar{I}=\frac{\left(1+2 M+M^{2}\right)}{\bar{\rho}_{j} \bar{a}_{j}}\left[\frac{p^{\prime 2}}{\rho_{r}^{2} a_{r}^{4}}\right] \rho_{r}^{2} a_{r}^{4}
$$

where $M$ is the Mach number of the flow.

The dimensionless quantity $\overline{p^{\prime 2}} /\left(\rho_{r}^{2} a_{r}^{4}\right)$ in equation (11) is computed by the nozzle flow numerical simulation code. At this time, broadband stochastic entropy wave input is not available for the computation. As a reasonable approximation, a time-periodic temperature wave train at a frequency of $250 \mathrm{~Hz}$ is used to simulate the entropy wave field coming out of the afterburner. The dimensionless pressure at the nozzle exit is measured over a long period of time. It is subsequently used to compute the quantity $\overline{p^{\prime 2}} /\left(\rho_{r}^{2} a_{r}^{4}\right)$. Our computation gives $\overline{p^{\prime 2}} /\left(\rho_{r}^{2} a_{r}^{4}\right)=0.945 \times 10^{-4}$ for a $15 \%$ temperature fluctuation inside the afterburner. At a temperature $1200 \mathrm{~K}$ and standard atmospheric pressure, the density of air is equal to $0.310 \mathrm{~kg} / \mathrm{m}^{3}$. 
But the pressure inside the afterburner is higher than the ambient pressure, with pressure ratio given equation (9). On accounting for the higher pressure inside the afterburner, it is found that $\rho_{r}=0.859 \mathrm{~kg} / \mathrm{m}^{3}$. By means of the above numerical values for the various quantities in formula (11), it is straightforward to find that the intensity level is,

$$
\text { Intensity Level (IL) }=10 \log \left(\frac{\bar{I}}{V_{\text {ref }}}\right)=175.5 \mathrm{~dB} \quad \text { where } I_{\text {ref }}=120 \mathrm{watt} / \mathrm{m}^{2}
$$

A noise intensity level of approximately $175.5 \mathrm{~dB}$ represents substantial sound radiation. This makes it likely that the new noise component of the F-22 aircraft is, indeed, indirect combustion noise.

\section{Summary and Conclusion}

This work is motivated by the question whether the dominant noise components of a high-performance aircraft at afterburner are the same as those of a heated supersonic laboratory jet. Our analysis of the F-22A jet noise data reveals clearly that they are quite different and that there is a new dominant noise component in addition to the well-known turbulent mixing noise components of a laboratory jet. For the F-22A aircraft, the new noise component is dominant in the angular directions between $110^{\circ}$ and $130^{\circ}$ and for frequencies above $200 \mathrm{~Hz}$. In addition, for several of the noise spectra examined, a low-frequency and relatively low-intensity unknown noise component is identified. This unknown noise component is easily identified for inlet angles $135^{\circ}$ and greater; it is a dominant noise component between $145^{\circ}$ and $150^{\circ}$.

In this study, we propose that the new noise components are most likely indirect combustion noise generated by the passage of entropy waves (hot spots) coming out of the afterburner through the nozzle of the jet engine. Unlike turbulent mixing noise that is generated by turbulence outside the nozzle, indirect combustion noise is an internal noise that radiates out from the nozzle exit. Because there are no measured flow and temperature fluctuation data, the proposed noise source cannot be readily verified experimentally. Therefore, numerical simulations are used to provide plausible support for the idea. However, further research is definitely needed to confirm the proposal.

In the literature, there are few publications on noise from high-performance aircraft operating at afterburner condition. It is our hope that this paper will serve to stimulate the interest of the jet noise research community on this class of problems. Clearly, in order to obtain a better understanding of the physical phenomenon and generation mechanism of this type of noise, engine scale measurements, laboratory scale experiments, numerical simulations and innovative theory are all very much needed.

\section{Acknowledgment}

The authors wish to thank the Office of Naval Research, grant monitor Dr. John Spyropoulous and technical representative Dr. Joseph Doychak for their support of this project (N00014-15-1-2008). 


\section{References}

[1] T.B. Neilsen, K.L. Gee and A.T. Wall, Similarity spectra analysis of high-performance jet aircraft noise. Journal of Acoustical Society of America 133 (2013) 2116-2125.

[2] K.L. Gee, V.W. Sparrow, M.M. James, J.M. Downing, C.M. Hobbs T.B. Gabrielson, and A.A. Atchley, The role of nonlinear effects in the propagation of noise from high-power jet aircraft. Journal of the Acoustical Society of America 123 (2008) 4082-4093.

[3] A.T. Wall, K.L. Gee, M.M. James,K.A. Bradley, S.A. McInerny, and T.B. Neilsen, Nearfield noise measurements of a high-performance military jet aircraft. Noise Control Engineering 60 (2012) 421-434.

[4] T.B. Neilsen, K.L. Gee, and M.M. James, Spectral characterization in the near-and mid-field of military jet aircraft noise. AIAA Paper 2013-2191.

[5] C.K.W. Tam, Supersonic jet noise. Annual Review of Fluid Mechanics 27 (1995) 17-43.

[6] C.K.W. Tam, M. Golebiowski, and J.M. Seiner, Two components of turbulent mixing noise from supersonic jets. AIAA Paper 96-1716 (1996).

[7] C.K.W. Tam, K. Viswanathan, K.K. Auhja, and J. Panda, The sources of jet noise: experimental evidence. Journal of Fluid Mechanics 615 (2008) 253-292.

[8] T. Suzuki, Coherent noise sources of a subsonic round jet investigated using hydrodynamic and acoustic phased-microphone arrays. Journal of Fluid Mechanics 730 (2013) 659-698.

[9] J.M. Seiner, M.K. Ponton, B.J. Jansen, and N.T. Lagen, The effects of temperature on supersonic jet noise emission. AIAA Paper 92-2046 (1992).

[10] K. Viswanathan, Aeroacoustics of hot jets. Journal of Fluid Mechanics 516 (2004) 39-82.

[11] C.K.W. Tam, Mach wave radiation from high-speed jets. AIAA Journal 47, 2440-2448.

[12] B.P. Petitjean, K. Viswanathan, and D.K. McLaughlin, Acoustic pressure waveforms measured in high speed jet noise experiencing nonlinear propagation. International Journal of Aeroacoustics 5 (2006) 193-215.

[13] C.K.W. Tam, and H.K. Tanna, Shock associated noise of supersonic jets from convergentdivergent nozzles. Journal of Sound and Vibration 81 (1982) 337-358.

[14] T.D. Norum, and J.M. Seiner, Measurements of static pressure and far field acoustics of shock containing supersonic jets. NASA TM 84521.

[15] J.R. Mahan and A. Karchmer, Combustion and core noise. in Aeroacoustics of Flight Vehicles: Theory and Practice, ed. Hubbard, H.H., NASA RP 12581 (1991) 483-517.

[16] C.K.W. Tam, N.N. Pastouchenko, J. Mendoza, and D. Brown, Combustion noise of auxiliary power units. AIAA Paper 2005-2829.

[17] B.T. Chu and L.S.G. Kovasznay, Non-linear interactions in a viscous heat-conducting compressible gas. Journal of Fluid Mechanics 3 (1958) 494-514.

[18] S.M. Candel, Analytical studies of some acoustic problems of jet engines. Ph.D. thesis, California Institute of Technology, Pasadena, California, 1972

[19] F.E. Marble and S.M. Candel, Acoustic disturbances from gas non-uniformities convected through a nozzle. Journal of Sound and Vibration 55 (1977) 225-243.

[20] C.L. Morfey, Amplification of aerodynamic noise by convected flow inhomogeneities. Journal of Sound and Vibration 31 (1971) 391-397.

[21] J.E. Ffowcs Williams, and M.S. Howe, The generation of sound by density inhomogeneities in low Mach number nozzle flows. Journal of Fluid Mechanics 70 (1975) 603 -622.

[22] N.A. Cumpsty, Jet engine combustion noise: pressure, entropy and vorticity perturbations produced by unsteady combustion and heat addition. Journal of Sound and Vibration $\mathbf{6 6}$ (1979) 527-544.

[23] N.A. Cumpsty and F.E. Marble, The interaction of entropy fluctuations with turbine blade rows: a mechanism of turbojet engine noise. Proceeding of the Royal Society of London, A 357 (1977) 323-344. 
[24] G.F. Pickett, Core Engine noise due to temperature fluctuations convecting through turbine blade rows. AIAA Paper 1975-528.

[25] Y. Sinai, The generation of combustion noise by chemical inhomogeneities in steady, lowMach number duct flows. Journal of Fluid Mechanics 99 (1980) 7-22.

[26] F. Bake, N. Kings, A. Fischer, and I. Rohle, Experimental investigation of the entropy noise mechanism in aero-Engines, International Journal of Aeroacoustics 8 (2009) 125-142.

[27] F. Bake, C. Richter, B. Muhlbauer, N. Kings, I. Rohle, F. Thiele, and B. Noll, The entropy wave generator: a reference case on entropy noise. Journal of Sound and Vibration 326 (2009) 574-598.

[28] M. Leyko, F. Nicoud, and T. Poinsot, Comparison of direct and indirect combustion noise mechanisms in a model combustor. AIAA Journal 47 (2009) 2709-2716.

[29] M.S. Howe, Indirect combustion noise. Journal of Fluid Mechanics 659 (2010) 267-288.

[30] C.S. Goh, A.S. Morgans, Phase prediction of the response of choked nozzles on entropy and acoustic disturbances. Journal of Sound and Vibration 330 (2011) 5184-5198.

[31] C.S. Goh, A.S. Morgans, The influence of entropy waves on the thermoacoustic stability of a model combustor. Combustion Science and Technology 185 (2013) 249-268.

[32] A. Giauque, M. Huet, and F. Clero, Analytical analysis of indirect combustion noise in subcritical nozzles. Journal of Engineering for Gas Turbines Power 134 (2012) 111202, 1-8.

[33] I. Duran, S. Moreau and T. Poinsot, Analytical and numerical study of combustion noise through a subsonic nozzle. AIAA Journal 51 (2013) 42-52.

[34] A. Mishra, and D.J. Bodony, Evaluation of actuator disk theory for predicting indirect combustion noise. Journal of Sound and Vibration 332 (2013) 821-838.

[35] A.S. Morgans, C.S. Goh, and J.A. Jahan, The dissipation and shear dispersion of entropy waves in combustor thermoacoustics. Journal of Fluid Mechanics 733-R2 (2013) 1-11.

[36] C.K.W. Tam, and S.A. Parrish, On the generation of indirect combustion noise. AIAA Paper 2014-3315.

[37] F.Q. Hu, A stable perfectly matched layer for linearized Euler equations in unsplit physical variables. Journal of Computational Physics 173 (2001) 455-480.

[38] F.Q. Hu, Absorbing boundary conditions. International Journal of Computational Fluid Dynamics 18 (2004) 513-522.

[39] C.K.W. Tam, TAM, Computational Aeroacoustics: a wavenumber approach, Cambridge University Press.

[40] C.K.W. Tam, and J.C. Webb, Dispersion-Relation-Preserving finite difference schemes for computational acoustics. Journal of Computational Physics 107, 262-281.

[41] C.K.W. Tam, J.M.Seiner, and J.C. Yu, Proposed relationship between broadband shock associated noise and screech tones. Journal of Sound and Vibration 110 (1986) 309-321.

[42] R.H. Cantrell and R.W. Hart, Interaction between sound and flow in acoustic cavities: mass, momentum and energy considerations. Journal of Acoustical Society of America 36 (1964) 697-706.

[43] C.L. Morfey Acoustic energy in non-uniform flows. Journal of Sound and Vibration 13 (1971) 159-170. 